\title{
On the Fundamental Mass-Period Functions of Extrasolar Planets
}

\author{
Ing-Guey Jiang ${ }^{a}$, Li-Chin Yeh $^{b}$, Yen-Chang Chang ${ }^{b}$, Wen-Liang Hung ${ }^{c}$ \\ ${ }^{a}$ Department of Physics and Institute of Astronomy, \\ National Tsing Hua University, Hsin-Chu, Taiwan \\ ${ }^{b}$ Department of Applied Mathematics, \\ National Hsinchu University of Education, Hsin-Chu, Taiwan \\ ${ }^{c}$ Graduate Institute of Computer Science, \\ National Hsinchu University of Education, Hsin-Chu, Taiwan
}

\begin{abstract}
Employing a catalog of 175 extrasolar planets (exoplanets) detected by the Dopplershift method, we constructed the independent and coupled mass-period functions. It is the first time in this field that the selection effect is considered in the coupled massperiod functions. Our results are consistent with those in Tabachnik and Tremaine (2002) with the major differences that we obtain a flatter mass function but a steeper period function. Moreover, our coupled mass-period functions show that about 2.5 percent of stars would have a planet with mass between Earth Mass and Neptune Mass, and about 3 percent of stars would have a planet with mass between Neptune Mass and Jupiter Mass $\left(M_{J}\right)$.
\end{abstract}

Key words: planetary systems, methods: data analysis, methods: numerical, methods: statistical

\section{Introduction}

The mass (size) function, i.e. the differential form for the number of objects as a function of mass (size),

$$
f_{M}(m)=\frac{d N}{d m}
$$

has been an important physical property to be investigated in many fields of astronomy such as galaxies, stars, asteroids and also dust grains. The importance lies in the meaning that this function 
is related with the formation and evolution of particular types of objects. Due to the fact that the mass function can be studied either through observational techniques or theoretical calculations, numerous research projects have been done on this subject.

For stars, the initial mass function (IMF) is the distribution of stellar masses from one star formation event in a given volume of space. Although the star-forming conditions vary with the environment, the measured IMF appears to be universal and can be modeled by a power-law,

$$
f_{M}(m)=\frac{d N}{d m}=c_{\star} m^{-\alpha_{\star}}
$$

where $c_{\star}$ is a normalization constant, and $\alpha_{\star}=2.35$ for the well-known Salpeter IMF. According to Kroupa, Tout, and Gilmore (1993),

$$
\left\{\begin{array}{l}
f_{M}(m)=c_{1} m^{-4.5} \text { for } m>1 M_{\odot}, \\
f_{M}(m)=c_{2} m^{-2.2} \text { for } 0.5 M_{\odot}<m<1 M_{\odot}, \\
f_{M}(m)=c_{3} m^{-1.2} \text { for } m<0.5 M_{\odot},
\end{array}\right.
$$

where $m$ is the star's mass, and $c_{1}, c_{2}, c_{3}$ are constants to be determined by the total number of stars in the considered system.

Moreover, there is a new development in astronomy that more than 300 planets are detected around solar type stars. The discovery has led to a new era of the study of planetary systems and thus triggered many interesting or controversial results of theoretical works (Jiang \& Ip 2001, Kinoshita \& Nakai 2001, Armitage et al. 2002, Ji et al. 2003, Boss 2005, Jiang \& Yeh 2007, Rice et al. 2008, Ji et al. 2009). For example, many discovered exoplanets have extremely short orbital periods. It is likely that they are formed at larger radial distances and migrate to the current locations later. However, because the migration timescale is too short, the rapid inward Type I migration caused by disc-core interactions poses a serious issue.

At the time when there were only about 70 detected exoplanets, Tabachnik \& Tremaine (2002) first used the maximum likelihood method to determine the mass and period functions with the assumption of two independent power laws. This work is, in fact, the only one that takes into account the selection effect and has intentions to obtain the fundamental mass-period functions. We have to note that different from the definition of stellar IMF, the planetary functions are constructed through the data of exoplanets from all different systems.

Without considering the selection effect, Zucker \& Mazeh (2002) calculated the linear correlation coefficient between mass and period and concluded that the mass-period correlation exists. 
The study of correlations within sub-groups in the cluster analysis done by Jiang et al. (2006) and Marchi (2007) also confirms the mass-period correlation. Thus, strictly speaking, the mass and period functions shall not be two independent power laws. Motivated by the above results, Jiang et al. (2007) employed an algorithm to generate a pair of positively correlated $\beta$-distributed random variables in order to construct a coupled mass-period function, and was the first one in the field of exoplanets to include the correlation into the construction of mass-period functions.

Moreover, through a nonparametric approach, Jiang et al. (2009) further constructed new coupled mass-period functions from 279 exoplanets and presented two main statistic results: (1) confirming the deficit of massive close-in planets; (2) discovering that more massive planets have larger ranges of possible semimajor axes. However, due to the fact that the selection effect is not considered in the above study, it is unclear how strong the statement (2) is. Yeh et al. (2009) argued that the planets larger than $1 M_{J}$ should be all within the detection limit, and thus implied that (2) should be a statistically valid statement.

Therefore, in order to address the above problem, it would be a great success if the coupled mass-period function can be obtained while the selection effect is considered simultaneously. It is this goal that motivates this work. The coupled mass-period function will be constructed by the same statistic method as in Jiang et al. (2009) and the selection effect would be considered through the similar procedure used in Tabachnik \& Tremaine (2002). However, as this work is an extension from Tabachnik \& Tremaine (2002), power laws would be employed as standard forms of mass-period functions. In order to consider the selection effect, only the exoplanets detected by the Doppler-shift method will be included as our samples.

We first constructed a reference-based catalog in $\S 2$, and discussed the normalization problem in $\S 3$. Then, the independent mass-period function was constructed in $\S 4$. In $\S 5$, mass-period correlations was studied. The coupled mass-period function was established in $\S 6$, and the fraction of stars with planets was discussed in $\S 7$. We concluded the paper in $\S 8$.

\section{A Reference-Based Catalog}

The most well-known catalog of exoplanets is the one maintained by Jean Schneider (Schneider Catalog hereafter), i.e. http://exoplanet.eu/catalog.php. This catalog is updated frequently when 
new detections are reported in refereed papers or conference proceedings. It is also notable that Butler et al.(2006a) updated the orbital solutions and compiled a list of 172 exoplanets. In order to extend the work of Tabachnik and Tremaine (2002) with more updated data, we here constructed a new exoplanet catalog, in which all samples were discovered by the Doppler-shift method, as listed in Appendix A. A major principle to construct this catalog is that all exoplanets in our catalog shall be reported as new detections in papers of refereed journals. In order to establish such a referencebased catalog, we searched and reviewed many published papers. We intended to make sure that these papers did report new discoveries, and to check which observational survey the results belong to. Thus, all the references listed in our catalog are the papers which reported new detections.

In our catalog, the first column is the data-set identity and the 2nd column is the name of the observational survey. The 3rd and 4th columns are the reference papers and the papers' corresponding identities. The 5th column lists the number of exoplanets discovered in that corresponding paper. Finally, column 6,7 , and 8 give the name, the projected mass $M$, and the orbital period $P$ of exoplanets. Most planets' $M$ and $P$ are obtained from Schneider Catalog. The values in Butler et al. (2006a) are used when they are missing in Schneider Catalog. We have to note that the exoplanet HD 154345b in Ref. (E-1) is removed here due to its extraordinarily larger period, 10900 days.

Besides, we found that some exoplanets were detected by more than one group around the same time, they could be reported as new discoveries in two different papers. We checked these and made a list in Table 2. However, they are still repeatedly listed in Appendix A, as our catalog was constructed based on published papers. Moreover, some papers studied more than one exoplanets, among which only one planet is a new discovery. In those papers, additional planets were included for a comparison purpose. We also carefully examined these kinds of papers, so that the reference papers in the catalog are exactly the papers which discover those listed exoplanets.

\section{The Normalization Problem}

In addition to the selection effect, the number of observed stars in a survey, $N_{s}$, is one of the key parameters to understand the probability that a star could host an exoplanet with particular orbital properties. Unfortunately, we found that the exact numbers of stars in surveys were not clearly mentioned normally. In most cases, an approximate number of target stars in a continuous 
long-term survey might be given, but the exact number of observed stars at that time when a new exoplanet detection was detected was usually not stated. For example, in Butler et al. (2003), it is written as "The Keck survey includes about 650 main-sequence and subgiant stars ...", and later in the same section, it is stated as "200 stars have one or more Keck observations but have been subsequently dropped from the program ...". In this case, neither 650 nor 450 can be used as the $N_{s}$ here.

Fortunately, Lineweaver and Grether (2003) obtained the numbers of target stars in surveys carefully and listed the results in their Table 4. In fact, they also estimated the number of repeated stars in different surveys, and finally determined the total number of target stars to be 1812 in these surveys. The numbers of detected planets in the corresponding surveys are also shown in their Table 3 , and the total number is 122 . Therefore, we decide to set $N_{\text {ratio }} \equiv N / N_{s} \equiv 122 / 1812=0.0673$ in our paper. This value of $N / N_{s}$ would be used when we determine the value of the normalization constant $c$ through the related equations, and also as a way to obtain $N_{s}$ in a particular survey for a given number of detected exoplanet $N$.

\section{Independent Mass-Period Functions}

In this section, the method and results based on the assumption of independent mass and period power-law functions will be described.

\subsection{The Method}

The procedures in this section, i.e. the analytical approach, the choice of parameters etc., follow exactly that of Tabachnik and Tremaine (2002). Here we describe the method in a selfconsistent way. However, please note that we simply consider the projected (minimum) mass $M$, which satisfies $M=M_{\text {real }} \sin i$, where $M_{\text {real }}$ is the real physical mass of the exoplanet and $i$ is the orbital inclination angle, in all calculations in this paper. That is, the mass $M$ in this paper means the minimum mass. The probability, $d p$, that a single star has an exoplanet with mass $M$ and orbital period $P$ in the range, $[M, M+d M],[P, P+d P]$, is given by the product of independent 
power laws on $M$ and $P$ as:

$$
d p=c\left[\frac{M}{M_{0}}\right]^{-\alpha}\left[\frac{P}{P_{0}}\right]^{-\beta} \frac{d M}{M} \frac{d P}{P},
$$

where $c, \alpha$ and $\beta$ are constants to be determined, and $M_{0}=1.5 M_{J}, P_{0}=90$ days. We assume that there are $N$ exoplanets in the data set, and let

$$
\left\{\begin{array}{l}
x_{i}=\ln \left(\frac{M_{i}}{M_{0}}\right) \\
y_{i}=\ln \left(\frac{P_{i}}{P_{0}}\right) \quad \text { for } \quad 1 \leq i \leq N
\end{array}\right.
$$

where $M_{i}, P_{i}$ are the mass and orbital period of one particular exoplanet. According to Eq.(2) and (6) of Tabachnik and Tremaine (2002), the value of $x_{i}-y_{i} / 3$ shall satisfy

$$
x_{i}-y_{i} / 3 \geq \ln \left[\frac{K_{D}}{28.4 \mathrm{~ms}^{-1}}\right]-\ln \left[\frac{M_{0}}{M_{J}}\right]+\frac{1}{3} \ln \left[\frac{P_{0}}{1 \mathrm{yr}}\right],
$$

where $K_{D}$ is the detection limit of the considered survey. For convenience, we define the right hand side of the above equation to be

$$
v_{D} \equiv \ln \left[\frac{K_{D}}{28.4 \mathrm{~ms}^{-1}}\right]-\ln \left[\frac{M_{0}}{M_{J}}\right]+\frac{1}{3} \ln \left[\frac{P_{0}}{1 \mathrm{yr}}\right] .
$$

The value of $v_{D}$ can be determined if the detection limit $K_{D}$ is exactly known. However, a range of possible values of $K_{D}$ is usually stated in a paper because it is related with the condition of instruments and weather. Similar to Tabachnik and Tremaine (2002), we set the smallest value of $x_{i}-y_{i} / 3$, i.e. $\min _{1 \leq i \leq N}\left\{x_{i}-y_{i} / 3\right\}$, in the considered data to be the value of $v_{D}$ and obtain the most likely $K_{D}$ reflected from the data through the above equation. Further, any value of $y_{i}$ shall satisfy

$$
y_{i} \leq \ln \left[\frac{P_{\max }}{P_{0}}\right],
$$

where $P_{\max }$ is the upper limit of orbital periods of detectable systems, which is proportional to the duration of the survey, as at least two orbits are required for a reliable detection. For convenience, we define

$$
u_{D} \equiv \ln \left[\frac{P_{\max }}{P_{0}}\right] \text {. }
$$

Similarly, it is not easy to obtain a consistent $P_{\max }$ from the published papers. We set the largest value of $y_{i}$ in the considered samples to be the value of $u_{D}$ and get the most likely $P_{\max }$ reflected from the data.

On the other hand, there are further constraints on $x_{i}$ and $y_{i}$. Let $M_{\max }$ be the maximum value of the mass in the data set for a considered survey, and define

$$
x_{\max }=\ln \left[\frac{M_{\max }}{M_{0}}\right],
$$


so we have a constraint on $x_{i}$ as

$$
x_{i} \leq x_{\max }
$$

Similarly, let $P_{\min }$ be the minimum value of the orbital period in the data set for a considered survey, and define

$$
y_{\min }=\ln \left[\frac{P_{\min }}{P_{0}}\right]
$$

we have another constraint on $y_{i}$ as

$$
y_{i} \geq y_{\min }
$$

Therefore, in the $x-y$ space, the exoplanet probability $d p$ can be expressed as:

$$
d p=c\left[\mathrm{e}^{x}\right]^{-\alpha}\left[\mathrm{e}^{y}\right]^{-\beta} d x d y,
$$

and the expected number of exoplanets in the area $d x d y$ in a survey of $N_{s}$ stars can be written as

$$
n(x, y) d x d y=N_{s} d p=c N_{s} \mathrm{e}^{-\alpha x} \mathrm{e}^{-\beta y} d x d y .
$$

Thus, as in Tabachnik and Tremaine (2002), the likelihood function $L$ is expressed as

$$
L=\Pi_{i=1}^{N} n\left(x_{i}, y_{i}\right) \exp \left[-\int_{D} n(x, y) d x d y\right],
$$

where the domain $D$ is $v+y / 3 \leq x \leq x_{\max }, y_{\min } \leq y \leq \tilde{u}$ with

$$
\left\{\begin{array}{l}
v=\min _{1 \leq i \leq N}\left\{x_{i}-\frac{y_{i}}{3}\right\} \\
u=\max _{1 \leq i \leq N}\left\{y_{i}\right\} \\
\tilde{u}=\min \left\{u, 3\left(x_{\max }-v\right)\right\}
\end{array}\right.
$$

Finally, through the maximum likelihood method, the values of $c, \alpha$, and $\beta$ can be determined by the following equations:

$$
\frac{\partial \ln L}{\partial c}=0, \quad \frac{\partial \ln L}{\partial \alpha}=0, \quad \text { and } \frac{\partial \ln L}{\partial \beta}=0 .
$$

The first one, $\partial \ln L / \partial c=0$, can be solved analytically and yielded an analytical expression of $c$ as shown in Eq. (16) of Tabachnik and Tremaine (2002). Using this analytical expression of $c$, $\partial \ln L / \partial \alpha=0$ and $\partial \ln L / \partial \beta=0$ can be solved numerically. 


\subsection{Individual Surveys}

First, the mass-period function is estimated through the data from one given particular survey. The previously mentioned equations are used to obtain the estimates of $\alpha, \beta$, and $c$. Their error bars are calculated as the standard deviations via the bootstrap algorithm with the replication size $B=2000$ (Jiang et al. 2007). The results are shown in Table 1, and each row is for one particular survey.

\section{Table 1.}

\begin{tabular}{|l|l|c|c|c|c|c|c|}
\hline Survey & Data Set & $\mathrm{N}$ & $\alpha$ & $\beta$ & $c \times 10^{3}$ & $\mathrm{~K}_{\mathrm{D}}\left(\mathrm{ms}^{-1}\right)$ & $\mathrm{P}_{\max }(\mathrm{yr})$ \\
\hline Lick & $(\mathrm{A})$ & 7 & $-0.401 \pm 1.051$ & $-0.144 \pm 0.388$ & $4.466 \pm 4.915$ & 19.794 & 14.296 \\
\hline Coralie & $(\mathrm{B})$ & 38 & $0.332 \pm 0.186$ & $-0.393 \pm 0.101$ & $2.969 \pm 0.372$ & 21.032 & 5.759 \\
\hline Elodie & $(\mathrm{C})$ & 14 & $-0.473 \pm 0.827$ & $-0.343 \pm 0.291$ & $2.327 \pm 5.876$ & 21.400 & 7.921 \\
\hline HARPS & $(\mathrm{D})$ & 23 & $0.081 \pm 0.146$ & $0.097 \pm 0.146$ & $1.733 \pm 4.189$ & 1.128 & 2.844 \\
\hline N2K & $(\mathrm{E})$ & 14 & $-0.071 \pm 0.425$ & $0.077 \pm 0.196$ & $3.320 \pm 4.057$ & 12.998 & 3.507 \\
\hline Keck & $(\mathrm{F})$ & 48 & $0.131 \pm 0.135$ & $-0.326 \pm 0.095$ & $1.362 \pm 0.243$ & 2.9304 & 9.184 \\
\hline AAPS & $(\mathrm{G})$ & 23 & $0.296 \pm 0.262$ & $-0.558 \pm 0.202$ & $1.778 \pm 0.715$ & 10.318 & 8.181 \\
\hline Other & $(\mathrm{H})$ & 17 & $0.168 \pm 0.350$ & $-0.341 \pm 0.192$ & $3.896 \pm 3.154$ & 28.189 & 5.957 \\
\hline Single & & 175 & $-0.143 \pm 0.040$ & $-0.124 \pm 0.044$ & $1.316 \pm 0.080$ & 1.128 & 14.296 \\
\hline
\end{tabular}

The data sets and total numbers of exoplanets used in the calculation of maximum likelihood method are shown in columns 2 and 3. The resulting values of $\alpha$ and $\beta$, i.e. the exponents of the power-law mass-period functions, are in columns 4 and 5. To make it clear, their values with error bars are plotted in Fig. 1. Table 1 shows that the Lick results have the largest error bars, due to the small numbers of planets. Note that Lick Survey has the smallest number of planets here. The reason is that we put the planets in Fischer et al.(2001) into Other Survey because both data came from Keck and Lick telescopes are used in that paper. We found that, among these surveys, the Coralie and Keck results have smaller error bars as Coralie Survey has 38 and Keck Survey has 48 planets. HARPS Survey has 23 planets, but the error bars are about as small as those in Coralie Survey due to the high precision on the measurement of radial velocities. In general, the absolute values of $\alpha$ are less than 0.5 , and the values of $\beta$ range from -0.6 to 0.1 . They both can be positive or negative. 
The final row is the result when all the above exoplanets are assumed to be discovered by a Single Imaginary Survey. However, as shown in Table 2, some planets are included in more than one data set. Although the summation of numbers of planets from all data sets is 184, there are actually 175 planets in total. The $\alpha$ and $\beta$ of the result of Single Imaginary Survey are both negative, and the corresponding error bars are very small due to a much larger number of samples.

Table 2. Repeated Exoplanets

\begin{tabular}{|l|l|l|}
\hline & Planet & Reference ID \\
\hline$(1)$ & HD102117 & $($ G-12) and (D-5) \\
\hline$(2)$ & HD 196050 & $($ B-1) and (G-2) \\
\hline$(3)$ & HD 216437 & $($ B-1) and (G-2) \\
\hline$(4)$ & HD 52265 & $($ B-4) and (F-16) \\
\hline$(5)$ & HD 192263 & $($ B-7) and (F-7) \\
\hline$(6)$ & HD 168443 c & $($ B-11) and (F-10) \\
\hline$(7)$ & HD 33636 & $($ C-2) and (F-9) \\
\hline$(8)$ & HD 37124 c & $($ F-1) and (F-12) \\
\hline$(9)$ & HD 92788 & $($ B-1) and (H-1) \\
\hline
\end{tabular}

\subsection{Multiple Surveys}

The analysis of individual surveys is generalized to Multiple Surveys here. In this analysis, different domains are considered for different surveys in Eq. (16). Thus, from Eq.(16), the likelihood function $L_{j}$ for the survey $j$ is

$$
L_{j}=\Pi_{i=1}^{N_{j}} n_{j}\left(x_{i, j}, y_{i, j}\right) \exp \left[-\int_{D_{j}} n_{j}(x, y) d x d y\right],
$$

where $N_{j}$ is the number of discovered planets for the survey $j$, and

$$
n_{j}(x, y)=c N_{s} e^{-\alpha x-\beta y}=\frac{c N_{j}}{N_{\text {ratio }}} e^{-\alpha x-\beta y}
$$

Thus, the log-likelihood is given by

$$
\ln L=\sum_{j=1}^{J} \ln L_{j}
$$


where $J$ is total number of considered surveys, and we have $J=8$ here. When $\ln L$ is maximized, the estimates for the parameters are obtained as:

$$
\begin{aligned}
& \alpha=-0.099 \pm 0.055 \\
& \beta=-0.129 \pm 0.040 \\
& c=(2.423 \pm 0.154) \times 10^{-3},
\end{aligned}
$$

where the error bars are also estimated via the bootstrap algorithm (Jiang et al. 2007). This set of $\alpha$ and $\beta$ with error bars are also shown in Fig. 1.

In order to show the distributions implied by these results and to be compared with the samples' histograms, we take integrations for the function $n(x, y)$, and thus the number of planets with masses between $M_{1}$ and $M_{2}$ and periods between $P_{1}$ and $P_{2}$ is given by

$$
\begin{aligned}
N_{\left[M_{1}, M_{2}\right][P 1, P 2]} & =\int_{\ln \left(P_{1} / P_{0}\right)}^{\ln \left(P_{2} / P_{0}\right)} \int_{\ln \left(M_{1} / M_{0}\right)}^{\ln \left(M_{2} / M_{0}\right)} n(x, y) d x d y=\iint c N_{s} e^{-\alpha x-\beta y} d x d y, \\
& =-\frac{c N_{s}}{\alpha} \int_{\ln \left(P_{1} / P_{0}\right)}^{\ln \left(P_{2} / P_{0}\right)}\left\{\left(\frac{M_{2}}{M_{0}}\right)^{-\alpha}-\left(\frac{M_{1}}{M_{0}}\right)^{-\alpha}\right\} e^{-\beta y} d y \\
& =\frac{c N_{s}}{\alpha \beta}\left\{\left(\frac{M_{2}}{M_{0}}\right)^{-\alpha}-\left(\frac{M_{1}}{M_{0}}\right)^{-\alpha}\right\}\left\{\left(\frac{P_{2}}{P_{0}}\right)^{-\beta}-\left(\frac{P_{1}}{P_{0}}\right)^{-\beta}\right\}
\end{aligned}
$$

After substituting the result of Single Imaginary Survey into the above equation, we obtain the expected number of planets in given mass or period intervals as shown in Fig. 2. With mass interval 0.5 Jupiter-mass and period interval 150 days, the crosses connected with dotted lines in Fig. 2 show the planetary distributions in mass space (see Fig. 2(a)), and also in period space (see Fig. 2(b)). For the comparison, the mass and period histograms of 175 exoplanets and the corresponding results derived from the Multiple Surveys are also plotted in Figs. 2(a)-(b).

\section{Mass-Period Correlation Coefficients}

In addition to the mass and period histograms and distributions shown in Fig. 2, the exoplanets' locations on the mass-period space, i.e. $M-P$ space, are often presented, as the mass and orbital period are the most important physical parameters of exoplanets. Fig. 3 is the distribution of 175 exoplanets in logarithmic space, $x-y$ space, where $x, y$ are as defined previously. The region enclosed by four solid lines is the Domain D, where all 175 planets are included. In order to investigate the strength of mass-period correlations, the Spearman rank-order correlation coefficients $\rho_{S}$ 
are calculated, as shown in Table 3.

Table 3.

\begin{tabular}{|l|c|c|c|}
\hline Survey & Data Set & $N$ & $\rho_{S}$ \\
\hline Lick & $(\mathrm{A})$ & 7 & 0.143 \\
\hline Coralie & $(\mathrm{B})$ & 38 & 0.351 \\
\hline Elodie & $(\mathrm{C})$ & 14 & 0.431 \\
\hline HARPS & $(\mathrm{D})$ & 23 & 0.564 \\
\hline N2K & $(\mathrm{E})$ & 14 & 0.552 \\
\hline Keck & $(\mathrm{F})$ & 48 & 0.532 \\
\hline AAPS & $(\mathrm{G})$ & 23 & 0.553 \\
\hline Other & $(\mathrm{H})$ & 17 & 0.542 \\
\hline Single & & 175 & 0.540 \\
\hline
\end{tabular}

In general, the values of $\rho_{S}$ are larger than 0.5 in five individual surveys and the Single Imaginary Survey of overall 175 planets. These results imply a strong positive correlation (Cohen 1988). Because there is a mass-period correlation, we shall consider the mass-period coupling and construct coupled mass-period functions in this paper.

On the other hand, as pointed in Jiang et al.(2006) and in Marchi (2007), the correlation of each group of exoplanets identified in the clustering analysis might be linked with the physical mechanisms more easily. The overall correlations could be more difficult to explain. A recent result by Marchi et al. (2009) is a good example that two dominated groups of close-in exoplanets are found and can be explained by two physical mechanisms successfully. This is an interesting and important topic that we would like to do further investigations in the future. However, the main point in this section is to re-confirm that there is indeed a mass-period correlation for our selected samples, and thus it is necessary to construct the coupled mass-period functions in this paper. 


\section{Coupled Mass-Period Functions}

We consider the mass-period coupling and construct coupled mass-period functions here. We define variables $x=\ln \left(M / M_{0}\right), y=\ln \left(P / P_{0}\right)$, and their probability density functions as:

$$
\begin{aligned}
& f_{X}(x)=\frac{\alpha e^{-\alpha x}}{e^{-\alpha x_{\min }}-e^{-\alpha x_{\max }}}, x_{\min } \leq x \leq x_{\max } \\
& f_{Y}(y)=\frac{\beta e^{-\beta y}}{e^{-\beta y_{\min }}-e^{-\beta y_{\max }}}, y_{\min } \leq y \leq y_{\max }
\end{aligned}
$$

Based on the copula modeling method introduced in Jiang et al. (2009), the coupled probability density function $f_{X Y}(x, y)$ is

$$
\begin{aligned}
& f_{X Y}(x, y)=\frac{\partial^{2} C\left(F_{X}(x), F_{Y}(y) ; \theta\right)}{\partial F_{X} \partial F_{Y}} f_{X}(x) f_{Y}(y) \\
= & \frac{-\theta\left(e^{-\theta}-1\right) e^{-\theta F_{X}(x)} e^{-\theta F_{Y}(y)}}{\left[e^{-\theta}-1+\left(e^{-\theta F_{X}(x)}-1\right)\left(e^{-\theta F_{Y}(y)}-1\right)\right]^{2}} f_{X}(x) f_{Y}(y),
\end{aligned}
$$

where the function $C\left(F_{X}(x), F_{Y}(y) ; \theta\right)$ is given by

$$
C\left(u_{1}, u_{2} ; \theta\right)=\frac{-1}{\theta} \ln \left[1+\frac{\left(e^{-\theta u_{1}}-1\right)\left(e^{-\theta u_{2}}-1\right)}{e^{-\theta}-1}\right],
$$

$u_{1}$ is the integral of $f_{X}(x)$, and $u_{2}$ is the integral of $f_{Y}(y)$. Thus,

$$
\begin{aligned}
& u_{1} \equiv F_{X}(x) \equiv \frac{e^{-\alpha x_{\min }}-e^{-\alpha x}}{e^{-\alpha x_{\min }}-e^{-\alpha x_{\max }}}, x_{\min } \leq x \leq x_{\max }, \\
& u_{2} \equiv F_{Y}(y) \equiv \frac{e^{-\beta y_{\min }}-e^{-\beta y}}{e^{-\beta y_{\min }}-e^{-\beta y_{\max }}}, y_{\min } \leq y \leq y_{\max }
\end{aligned}
$$

The dependence parameter $\theta(-\infty<\theta<\infty)$ can be positive, zero and negative, corresponding to the positive dependence, independence and negative dependence between two variables $x$ and $y$, respectively. When $\theta$ approaches to zero, the term $\partial^{2} C / \partial F_{X} \partial F_{Y}$ would approach to one, and $f_{X Y}(x, y)=f_{X}(x) f_{Y}(y)$. Thus, $\partial^{2} C / \partial F_{X} \partial F_{Y}$ is called the coupling factor in this paper as it controls the $x-y$ dependence.

The function $C\left(u_{1}, u_{2} ; \theta\right)$ in Eq. (28) is called the Frank copula function. In fact, there are many available copula functions, and the reason why we choose this one is that it is more flexible as it allows to have negative, zero, and positive correlations.

Further, the expected number of exoplanets in the area $d x d y$ in a survey of $N_{s}$ stars is

$$
n(x, y) d x d y=c N_{s} f_{X Y}(x, y) d x d y
$$


where the parameter $c$ is a constant to be determined. For a given function $n(x, y)$, the number of planets with masses between $M_{1}$ and $M_{2}$ and periods between $P_{1}$ and $P_{2}$ is determined by

$$
\begin{aligned}
N_{\left[M_{1}, M_{2}\right][P 1, P 2]} & =\int_{\ln \left(P_{1} / P_{0}\right)}^{\ln \left(P_{2} / P_{0}\right)} \int_{\ln \left(M_{1} / M_{0}\right)}^{\ln \left(M_{2} / M_{0}\right)} n(x, y) d x d y \\
& =c N_{s} \int_{\ln \left(P_{1} / P_{0}\right)}^{\ln \left(P_{2} / P_{0}\right)} \int_{\ln \left(M_{1} / M_{0}\right)}^{\ln \left(M_{2} / M_{0}\right)} f_{X Y}(x, y) d x d y .
\end{aligned}
$$

On the other hand, the likelihood function is

$$
\begin{aligned}
L & =\prod_{i=1}^{N} n\left(x_{i}, y_{i}\right) \exp \left[-\int_{D} n(x, y) d x d y\right] \\
& =\prod_{i=1}^{N} c N_{s} f_{X Y}\left(x_{i}, y_{i}\right) \exp \left[-c N_{s} \int_{y_{\min }}^{\tilde{u}} \int_{v+y / 3}^{x_{\max }} f_{X Y}(x, y) d x d y\right]
\end{aligned}
$$

After the derivation shown in Appendix $\mathrm{B}, \ln L$ is finally expressed as a function of $\alpha, \beta$, and $\theta$. The maximum likelihood method is used to simultaneously estimate the parameters $\alpha, \beta$ and $\theta$ through the full $\log$ likelihood $\ln L$. The estimates of $c, \alpha, \beta$ and $\theta$ for each survey are listed in Table 4. Moreover, as the procedure in $\S 4$, here we also generalize the result to the case of Multiple Surveys and the result is at the bottom of Table 4. The bootstrap method is also used to get error bars. Fig. 4 shows the values of $\alpha$ and $\beta$ with error bars. The result of Single Imaginary Survey of 175 planets gives $\alpha=-0.187 \pm 0.034, \beta=-0.133 \pm 0.041$, so the mass (period) function has a power-index $-0.813 \pm 0.034(-0.867 \pm 0.041)$. Moreover, the result of Multiple Surveys gives $\alpha=-0.038 \pm 0.080, \beta=-0.137 \pm 0.044$, and thus the mass (period) power-index is $-0.962 \pm 0.080$ $(-0.863 \pm 0.044)$. On the other hand, the mass (period) power-index obtained in Tabachnik and Tremaine $(2002)$ is $-1.11 \pm 0.10(-0.73 \pm 0.06)$. Thus, considering the error bars, our results are consistent with those in Tabachnik and Tremaine (2002). However, our mean values imply a flatter mass function but a slightly steeper period function. We hope to use the future data to investigate whether this new result remains to be valid.

In Fig. 5(a), from the result of Multiple Surveys, the coupled probability density function in $x-y$ space, $f_{X Y}(x, y)$, is shown as a three-dimensional plot. The corresponding contour is in Fig. 5(b). To visualize it in a realistic space, the above two are transformed to be in $M-P$ space and shown in Figs. 6(a)-(b), where the coupled probability density function in $M-P$ space, $f_{M P}(M, P)$ is defined by

$$
f_{M P}(M, P)=f_{X Y}(x, y)|J|,
$$


where $J$ is the Jacobian determinant $\partial x / \partial M \times \partial y / \partial P$. As we know from Eq.(27), the massperiod coupling is primarily determined by the coupling factor. In order to visualize it, the threedimensional and color contour plots in $x-y$ space are shown in Figs. 7(a)-(b). It shows that the coupling factor is bigger when $x$ and $y$ have the same sign, i.e. both positive or both negative. This implies that each group of exoplanets would have its own strength of mass-period correlations if the exoplanets are clustered into a few groups. For example, Marchi et al. (2009) did the clustering analysis on exoplanets, and found the existence of two types of close-in planets. They discovered that these two types of planets have very different distributions of semi-major axes. Moreover, Marchi et al. (2009) also proposed possible mechanisms to produce these two different types of exoplanets.

Figs. 8(a)-(b) show the coupled mass-period probability density function (pdf), $f_{X Y}(x, y)$, for a few given masses or periods. For the purpose of comparison, the pdfs without coupling factor, i.e. $f_{X}(x) \times f_{Y}(y)$ are also plotted in Figs. 8(c)-(d). In Fig. 8(a) and Fig. 8(c), the solid curve is for $x=\ln \left(1 M_{J} / M_{0}\right)$, the dotted curve is for $x=\ln \left(5 M_{J} / M_{0}\right)$, the short dashed curve is for $x=\ln \left(10 M_{J} / M_{0}\right)$, and the long dashed curve is for $x=\ln \left(15 M_{J} / M_{0}\right)$. In Fig. 8(b) and Fig. 8(d), the solid curve is for $y=\ln \left(1\right.$ day $\left./ P_{0}\right)$, the dotted curve is for $y=\ln \left(50\right.$ days $\left./ P_{0}\right)$, the short dashed curve is for $y=\ln \left(100\right.$ days $\left./ P_{0}\right)$, and the long dashed curve is for $y=\ln \left(150\right.$ days $\left./ P_{0}\right)$. In general, it shows that the coupling factor does change the shapes of mass and period functions. To be more realistic, the curves in Fig. 8 are re-plotted as functions of $M$ and $P$ as shown in Fig. 9. 
Table 4.

\begin{tabular}{|l|c|c|c|c|c|c|}
\hline Survey & Data Set & $\mathrm{N}$ & $\alpha$ & $\beta$ & $\theta$ & $c \times 10^{2}$ \\
\hline Lick & $(\mathrm{A})$ & 7 & $0.531 \pm 1.047$ & $-0.124 \pm 0.334$ & $2.289 \pm 5.201$ & $7.520 \pm 6.044$ \\
\hline Coralie & $(\mathrm{B})$ & 38 & $0.314 \pm 0.158$ & $-0.344 \pm 0.096$ & $0.963 \pm 1.438$ & $10.101 \pm 1.681$ \\
\hline Elodie & $(\mathrm{C})$ & 14 & $-0.455 \pm 0.565$ & $-0.203 \pm 0.233$ & $4.757 \pm 2.978$ & $6.878 \pm 11.234$ \\
\hline HARPS & $(\mathrm{D})$ & 23 & $0.046 \pm 0.193$ & $0.092 \pm 0.168$ & $1.831 \pm 1.863$ & $7.013 \pm 0.312$ \\
\hline N2K & $(\mathrm{E})$ & 14 & $0.045 \pm 0.438$ & $0.130 \pm 0.191$ & $1.795 \pm 2.045$ & $7.049 \pm 1.621$ \\
\hline Keck & $(\mathrm{F})$ & 48 & $0.019 \pm 0.132$ & $-0.217 \pm 0.082$ & $3.957 \pm 1.521$ & $8.145 \pm 0.882$ \\
\hline AAPS & $(\mathrm{G})$ & 23 & $0.018 \pm 0.220$ & $-0.416 \pm 0.139$ & $6.786 \pm 2.288$ & $7.337 \pm 1.353$ \\
\hline Other & $(\mathrm{H})$ & 17 & $-0.069 \pm 0.402$ & $-0.229 \pm 0.182$ & $3.970 \pm 2.511$ & $7.166 \pm 1.635$ \\
\hline Single & & 175 & $-0.187 \pm 0.034$ & $-0.133 \pm 0.041$ & $4.848 \pm 0.779$ & $6.832 \pm 0.098$ \\
\hline Multiple & & 184 & $-0.038 \pm 0.080$ & $-0.137 \pm 0.044$ & $2.973 \pm 0.639$ & $7.378 \pm 0.289$ \\
\hline
\end{tabular}

\section{The Fraction of Stars with Planets}

From both theoretical and observational points of view, it is important to know what fraction of stars would have planets. If we divide $N_{\left[M_{1}, M_{2}\right][P 1, P 2]}$ by $N_{s}$ in Eq. (24), the probability that a star could host a planet with masses between $M_{1}$ and $M_{2}$ and periods between $P_{1}$ and $P_{2}$ is given by

$$
\text { Prob }=\frac{c}{\alpha \beta}\left\{\left(\frac{M_{2}}{M_{0}}\right)^{-\alpha}-\left(\frac{M_{1}}{M_{0}}\right)^{-\alpha}\right\}\left\{\left(\frac{P_{2}}{P_{0}}\right)^{-\beta}-\left(\frac{P_{1}}{P_{0}}\right)^{-\beta}\right\}
$$

for the independent mass-period function.

As the power indexes obtained from the results of Single Imaginary Survey are different from those from Multiple Surveys, we calculated both results using Eq. (35). We used the symbol $\operatorname{Prob}_{S}$ to represent the results of Single Imaginary Survey and used $\operatorname{Prob}_{M}$ for the results of Multiple Surveys.

On the other hand, for coupled mass-period functions, we have

$$
\begin{aligned}
& \operatorname{Prob}_{C}=\frac{1}{N_{s}} \int_{\ln \left(P_{1} / P_{0}\right)}^{\ln \left(P_{2} / P_{0}\right)} \int_{\ln \left(M_{1} / M_{0}\right)}^{\ln \left(M_{2} / M_{0}\right)} n(x, y) d x d y=\iint c f_{X Y}(x, y) d x d y, \\
= & c \iint \frac{-\theta\left(e^{-\theta}-1\right) e^{-\theta F_{X}(x)} e^{-\theta F_{Y}(y)}}{\left[e^{-\theta}-1+\left(e^{-\theta F_{X}(x)}-1\right)\left(e^{-\theta F_{Y}(y)}-1\right)\right]^{2}} f_{X}(x) f_{Y}(y) d x d y .
\end{aligned}
$$


Similarly, we used the symbol $\operatorname{Prob}_{C S}$ to represent the results of Single Imaginary Survey and used $\operatorname{Prob}_{C M}$ for the results of Multiple Surveys in the case of coupled mass-period functions.

Tabachnik \& Tremaine (2002) estimated the expected number of planets per star for given period and mass range through their best results of multiple surveys, which is in fact equivalently defined as $\operatorname{Prob}_{M}$ here. When $M_{1}=M_{J}, M_{2}=10 M_{J}, P_{1}=2$ days, and $P_{2}=10$ yrs $=3650$ days, Tabachnik \& Tremaine (2002) found $\operatorname{Prob}_{M}=0.036$ and concluded that 4 percent of solar-type stars have a planet in the above ranges. For the same given ranges, we obtain: Prob $_{S}=0.02618$, $\operatorname{Prob}_{M}=0.04667, \operatorname{Prob}_{C S}=0.02909$, and $\operatorname{Prob}_{C M}=0.02273$. Thus, our results of Multiple Surveys are similar to those in Tabachnik \& Tremaine (2002). Moreover, the estimated probabilities from the coupled mass-period functions are smaller but still consistent with those in Tabachnik \& Tremaine (2002). In Tabachnik \& Tremaine (2002), the case with $M_{1}=0.003 M_{J}$ (i.e. Earth Mass), $M_{2}=10 M_{J}, P_{1}=2$ days, and $P_{2}=10 \mathrm{yrs}=3650$ days is also estimated and has a probability as 0.18. However, our results show that: $\operatorname{Prob}_{S}=0.06406$. $\operatorname{Prob}_{M}=0.1264, \operatorname{Prob}_{C S}=0.06492$, and $\operatorname{Prob}_{C M}=0.0736$. Note that the result with $M_{1}=0.003 M_{J}$ (i.e. Earth Mass) is an extrapolation as this small mass is out of the mass range of 175 samples. Under the assumption that the smaller planets shall follow the trend of our mass-period function, and the fact that the Earth-Mass planet does exist in our universe, i.e. Solar System, this extrapolation shall lead to a good estimation.

On the other hand, Naef et al. (2005) also gave estimations about the fractions of star with planets more massive than $0.5 M_{J}$ within three periods: 0.7 percent for period $<5$ days, 4 percent for period $<1500$ days, and 7.3 percent for period $<3900$ days. Correspondingly, using our samples and equations here, for $M_{1}=0.5 M_{J}, M_{2}=M_{\max }, P_{1}=P_{\min }$, and $P_{2}=5$ days (Note that, in 175 samples, the smallest mass $M_{\min }=0.0158$, the largest mass $M_{\max }=18.39$, the smallest period $P_{\min }=1.328$, the largest period $P_{\max }=5218$, and the units are $M_{J}$ and days), we obtained the fraction of stars with planets to be less than 1 percent. For $M_{1}=0.5 M_{J}, M_{2}=M_{\max }, P_{1}=P_{\min }$, and $P_{2}=1500$ days, we obtained the fraction of stars with planets to be about 3 to 6 percent. Moreover, for $M_{1}=0.5 M_{J}, M_{2}=M_{\max }, P_{1}=P_{\min }$, and $P_{2}=3900$ days, the results are about 4 to 8 percent.

Finally, we are interested in the possibility to have a planet with mass between Earth Mass and Neptune Mass, for any period, so we set $M_{1}=0.003, M_{2}=0.05, P_{1}=P_{\min }$, and $P_{2}=P_{\max }$, and get: $\operatorname{Prob}_{S}=1.604 \%, \operatorname{Prob}_{M}=3.649 \%, \operatorname{Prob}_{C S}=1.263 \%$, and $\operatorname{Prob}_{C M}=2.542 \%$. We are 
also interested in the possibility between Neptune Mass and Jupiter Mass, for any possible period, thus we set $M_{1}=0.05, M_{2}=1, P_{1}=P_{\text {min }}$, and $P_{2}=P_{\text {max }}$, and obtain: Prob $_{S}=2.589 \%$, Prob $_{M}$ $=5.182 \%, \operatorname{Prob}_{C S}=2.319 \%$, and $\operatorname{Prob}_{C M}=3.021 \%$. All the above mentioned results calculated by the equations in this paper are listed in Table 5 .

Table 5 .

\begin{tabular}{|c|c|c|c|c|c|c|c|c|}
\hline Case & $M_{1}$ & $M_{2}$ & $P_{1}$ & $P_{2}$ & Prob $_{S}$ & Prob $_{M}$ & Prob $_{C S}$ & Prob $_{C M}$ \\
\hline 1 & 1. & 10. & 2. & 3650. & 0.02618 & 0.04667 & 0.02909 & 0.02273 \\
\hline 2 & 0.003 & 10. & 2. & 3650. & 0.06406 & 0.12640 & 0.06492 & 0.0736 \\
\hline 3 & 0.5 & 18.39 & 1.328 & 5. & 0.00453 & 0.00790 & 0.00154 & 0.00169 \\
\hline 4 & 0.5 & 18.39 & 1.328 & 1500. & 0.03527 & 0.06255 & 0.03095 & 0.02661 \\
\hline 5 & 0.5 & 18.39 & 1.328 & 3900. & 0.04290 & 0.07631 & 0.04196 & 0.03658 \\
\hline 6 & 0.003 & 0.05 & 1.328 & 5218.0 & 0.01604 & 0.03649 & 0.01263 & 0.02542 \\
\hline 7 & 0.05 & 1. & 1.328 & 5218.0 & 0.02589 & 0.05182 & 0.02319 & 0.03021 \\
\hline
\end{tabular}

\section{Concluding Remarks}

In this paper, several steps have been taken to establish the fundamental mass-period functions of exoplanets. First of all, by reading a great number of published papers, we constructed a reference-based catalog of 175 exoplanets, which were all discovered through the Doppler-shift method. Employing this catalog, we determine the independent mass-period functions for individual surveys and also for the case of Multiple Surveys. Moreover, the coupled mass-period functions are also constructed for both individual and Multiple Surveys. The selection effects of surveys are considered in all the results, and thus it is the first time in this field that the selection effect is considered in the coupled mass-period functions. Our results are consistent with those in Tabachnik and Tremaine (2002) with the main differences that our results imply a flatter mass functions but a steeper period function.

On the other hand, our coupled mass-period functions are used to predict the possible fractions of stars in given mass and period ranges. Our results are consistent with previous works, and show that about 2.5 percent of stars would have a planet with mass between Earth Mass and Neptune Mass, and about 3 percent of stars would have a planet with mass between Neptune Mass and 
Jupiter Mass.

\section{Acknowledgment}

We thank the anonymous referee for useful suggestions that improved the paper enormously. We also owe a debt of thanks to Scott Tremaine for the communications about his paper, Tabachnik and Tremaine (2002), and the positive remarks on our paper. We are grateful to the National Center for High-performance Computing for computer time and facilities. This work is supported in part by the National Science Council, Taiwan.

\section{REFERENCES}

Armitage, P. J., et al. 2002, MNRAS, 334, 248

Bonfils, X., et al. 2005, A\&A, 443, L15

Bonfils, X., et al. 2007, A\&A, 474, 293

Boss, A. P. 2005, ApJ, 629, 535

Butler, R.P., et al. 1997, ApJ, 575, L115

Butler, R.P., et al. 1998, PASP, 110, 1389

Butler, R.P., et al. 1999, ApJ, 526, 916

Butler, R.P., et al. 2000, ApJ, 545, 504

Butler, R.P., et al. 2001, ApJ, 555, 410

Butler, R.P., et al. 2003, ApJ, 582, 455

Butler, R.P., et al. 2004, ApJ, 617, 580

Butler, R.P., et al. 2006a, ApJ, 646, 505

Butler, R.P., et al. 2006b, PASP, 118, 1685

Carter, B.D., et al. 2003, ApJ, 593, L43 
Cochran, W.D., et al. 1997, ApJ, 483, 457

Cohen, J, 1988, Statistical power analysis for the behavioral sciences, Lawrence Erlbaum Associates, New Jersey.

Correia, A.C.M., et al. 2005, A\&A, 440, 751

da. Silva, R., et al. 2006, A\&A, 446, 717

Delfosse, X., et al. 1998, A\&A, 338, L67

Eggenberger, A., et al. 2006, A\&A, 447, 1159

Fischer, D., et al. 1999, PASP, 755, 50

Fischer, D., et al. 2001, ApJ, 551, 1107

Fischer, D., et al. 2002a, ApJ, 564, 1028

Fischer, D., et al. 2002b, PASP,114, 529

Fischer, D., et al. 2003, ApJ, 586, 1394

Fischer, D., et al. 2005, ApJ, 620, 481

Fischer, D., et al. 2006, ApJ, 637, 1094

Fischer, D., et al. 2007, ApJ, 669, 1336

Galland, F., et al. 2005, A\&A, 444, L21

Ji, J., et al. 2003, ApJ, 585, L139

Ji, J., et al. 2009, RAA, 9, 703

Jiang, I.-G., Ip, W.-H. 2001, A\&A, 367,943

Jiang, I.-G., et al. 2006, MNRAS, 370, 1379

Jiang, I.-G., Yeh, L.-C. 2007, ApJ, 656, 534

Jiang, I.-G., et al. 2007, AJ, 134, 2061 
Jiang, I.-G., et al. 2009, AJ, 137, 329

Johnson, J.A., et al. 2006, ApJ, 647, 600

Johnson, J.A., et al. 2007, ApJ, 670, 833

Johnson, J.A., et al. 2008, ApJ, 675, 784

Jones, H.R., et al. 2002a, MNRAS, 333, 871

Jones, H.R., et al. 2002b, MNRAS, 337, 1170

Jones, H.R., et al. 2003, MNRAS, 341, 948

Jones, H.R., et al. 2006, MNRAS, 369, 249

Kinoshita, H., Nakai, H. 2001, PASJ, 53, L25

Korzennik, S.G., et al. 2000, ApJ, 533, L147

Kroupa, P., Tout, C., Gilmore, G. 1993, MNRAS, 262, 545

Lineweaver, C. H., Grether, D., 2003, ApJ, 598, 1350

Lo Curto, G., et al. 2006, A\&A, 451, 345

Lovis, C., et al. 2005, A\&A, 437, 1121

Lovis, C., et al. 2006, Nature, 441, 305

Marchi, S. 2007, ApJ, 666, 475

Marchi, S., et al. 2009, MNRAS, 394, L93

Marcy, G.W., et al. 1999, ApJ, 520, 239

Marcy, G.W., et al. 2000, ApJ, 536, L34

Marcy, G.W., et al. 2001a, ApJ, 555, 418

Marcy, G.W., et al. 2001b, ApJ, 556, 296

Marcy, G.W., et al. 2002, ApJ, 581,1375 
Marcy, G. W., et al 2005, ApJ, 619, 570

Mayor, M. \& Queloz, D. 1995, Nature, 378, 355

Mayor, M., et al. 2004, A\&A, 415, 391

Melo, C., et al. 2007, A\&A, 467, 721

Moutou, C., et al. 2005, A\&A, 439, 367

Moutou, C., et al. 2006, A\&A, 458, 327

Naef, D., et al. 2001a, A\&A, 375, 205

Naef, D., et al. 2001b, A\&A, 375, L27

Naef, D., et al. 2003, A\&A, 410, 1051

Naef, D., et al. 2004, A\&A, 414, 350

Naef, D., et al. 2005, Proceedings of the 13th Cambridge Workshop on Cool Stars, Stellar Systems and the Sun, held 5-9 July, 2004 in Hamburg, Germany, page 833, astro-ph/0409230v2

Naef, D., et al. 2007, A\&A, 470, 721

O’Toole, S.J., et al. 2007, ApJ, 660, 1636

Pepe, F., et al. 2002, A\&A, 388, 632

Pepe, F., et al. 2004, A\&A, 423, 385

Pepe, F., et al. 2007, A\&A, 462, 769

Perrier, C., et al. 2003, A\&A, 410, 1039

Queloz, D., et al. 2000, A\&A, 354, 99

Rivera, E.J., et al. 2005, ApJ, 634, 625

Rice, W. K. M., et al. 2008, MNRAS, 384, 1242

Robinson, S. E., et al. 2007, ApJ, 670, 1391 
Santos, N.C., et al. 2000, A\&A, 356, 599

Santos, N.C., et al. 2001, A\&A, 379, 999

Santos, N.C., et al. 2002, A\&A, 392, 215

Santos, N.C., et al. 2004, A\&A, 426, L19

Santos, N.C., et al. 2007, A\&A, 474, 647

Sato, B., et al. 2005, ApJ, 633,465

Sozzetti, A., et al. 2006, A\&A, 449, 417

Tabachnik, S., Tremaine, S. 2002, MNRAS, 335, 151

Tamuz, O., et al. 2008, A\&A, 480, L33

Tinney, C.G., et al. 2001, ApJ, 551, 507

Tinney, C.G., et al. 2002, ApJ, 571, 528

Tinney, C.G., et al. 2003, ApJ, 587, 423

Tinney, C.G., et al. 2005, ApJ, 623, 1171

Tinney, C.G., et al. 2006, ApJ, 647, 594

Udry, S., et al. 2000, A\&A 356, 590

Udry, S., et al. 2002, A\&A, 390, 267

Udry, S., et al. 2003, A\&A, 407, 679

Udry, S., et al. 2006, A\&A, 447, 361

Udry, S., et al. 2007, A\&A, 469, L43

Vogt, S.S., et al. 2000, ApJ, 536, 902

Vogt, S.S., et al. 2002, ApJ, 568, 352

Vogt, S.S., et al. 2005, ApJ, 632, 638 
Wright, J.T., et al. 2007, ApJ, 657, 533

Yeh, L.-C., et al. 2009, Proceedings of the 10th Asian-Pacific Regional IAU Meeting, page 80, China Science \& Technology Press, Beijing

Zucker, S., Mazeh, T., 2002, ApJ, 568, L113

Zucker, S., et al. 2004, A\&A, 426, 695

This preprint was prepared with the AAS LATEX macros v5.0. 


\section{Appendix A}

\begin{tabular}{|c|c|c|c|c|c|c|c|}
\hline Data Set & Survey & Reference & Ref. ID & $\mathrm{N}$ & Planet & $M$ & $P$ \\
\hline \multirow[t]{7}{*}{$(\mathrm{A})$} & \multirow[t]{7}{*}{ Lick } & Marcy et al.(2002) & $(\mathrm{A}-1)$ & 1 & $55 \mathrm{Cnc} \mathrm{d}$ & 3.835 & 5218 \\
\hline & & Fischer et al.(2002a) & $(\mathrm{A}-2)$ & 1 & 47 Uma c & 1.34 & 2594 \\
\hline & & \multirow[t]{3}{*}{ Butler et al.(1997) } & \multirow[t]{3}{*}{$(\mathrm{A}-3)$} & \multirow[t]{3}{*}{3} & $55 \mathrm{Cnc} \mathrm{b}$ & 0.824 & 14.65162 \\
\hline & & & & & Tau Boo b & 3.9 & 3.3135 \\
\hline & & & & & Ups And b & 0.69 & 4.61708 \\
\hline & & \multirow[t]{2}{*}{ Johnson et al.(2008) } & \multirow[t]{2}{*}{$(\mathrm{A}-4)$} & \multirow[t]{2}{*}{2} & Kappa CrB b & 1.8 & 1191 \\
\hline & & & & & HD 167042 b & 1.6 & 416.1 \\
\hline \multirow[t]{23}{*}{$(\mathrm{B})$} & \multirow[t]{23}{*}{ Coralie } & \multirow[t]{16}{*}{ Mayor et al. (2004) } & \multirow[t]{16}{*}{$(\mathrm{B}-1)$} & \multirow[t]{16}{*}{16} & HD 19994 & 2 & 454 \\
\hline & & & & & HD 65216 & 1.21 & 613.1 \\
\hline & & & & & HD 92788 & 3.86 & 377.7 \\
\hline & & & & & HD 111232 & 6.8 & 1143 \\
\hline & & & & & HD 114386 & 0.99 & 872 \\
\hline & & & & & HD 142415 & 1.62 & 386.3 \\
\hline & & & & & HD 147513 & 1 & 540.4 \\
\hline & & & & & HD 196050 & 3 & 1289 \\
\hline & & & & & HD 216437 & 2.1 & 1294 \\
\hline & & & & & HD 216770 & 0.65 & 118.45 \\
\hline & & & & & HD 6434 & 0.48 & 22.09 \\
\hline & & & & & HD 121504 & 0.89 & 64.6 \\
\hline & & & & & HD $83443 \mathrm{~b}$ & 0.4 & 2.985625 \\
\hline & & & & & HD $82943 \mathrm{~b}$ & 1.75 & 441.2 \\
\hline & & & & & HD $82943 \mathrm{c}$ & 2.01 & 219 \\
\hline & & & & & HD $169830 \mathrm{c}$ & 4.04 & 2102 \\
\hline & & \multirow[t]{2}{*}{ Tamuz et al. (2008) } & \multirow[t]{2}{*}{$(\mathrm{B}-2)$} & \multirow[t]{2}{*}{2} & HD 4113 & 1.56 & 526.62 \\
\hline & & & & & HD 156846 & 10.45 & 359.51 \\
\hline & & Udry et al.(2003) & $(\mathrm{B}-3)$ & 1 & HD 73256 & 1.87 & 2.54858 \\
\hline & & \multirow[t]{3}{*}{ Naef et al.(2001a) } & \multirow[t]{3}{*}{$(\mathrm{B}-4)$} & \multirow[t]{3}{*}{3} & GJ 3021 & 3.32 & 133.82 \\
\hline & & & & & HD 52265 & 1.13 & 118.96 \\
\hline & & & & & HD 169830 b & 2.88 & 225.62 \\
\hline & & Queloz et al.(2000) & $(\mathrm{B}-5)$ & 1 & GJ 86 & 4.01 & 15.766 \\
\hline
\end{tabular}




\begin{tabular}{|c|c|c|c|c|c|c|c|}
\hline Data Set & Survey & Reference & Ref. ID & $\mathrm{N}$ & Planet & $M$ & $P$ \\
\hline \multirow[t]{15}{*}{ (B) } & \multirow[t]{15}{*}{ Coralie } & \multirow[t]{2}{*}{ Udry et al(2000) } & \multirow[t]{2}{*}{$(\mathrm{B}-6)$} & \multirow[t]{2}{*}{2} & HD 75289 & 0.42 & 3.51 \\
\hline & & & & & HD 130322 & 1.08 & 10.724 \\
\hline & & Santos et al.(2000) & $(\mathrm{B}-7)$ & 1 & HD 192263 & 0.72 & 24.348 \\
\hline & & \multirow[t]{2}{*}{ Santos et al.(2001) } & \multirow[t]{2}{*}{$(\mathrm{B}-8)$} & \multirow[t]{2}{*}{2} & HD 28185 & 5.7 & 383 \\
\hline & & & & & HD 213240 & 4.5 & 951 \\
\hline & & Correia et al.(2005) & $(\mathrm{B}-9)$ & 1 & HD $202206 \mathrm{c}$ & 2.44 & 1383.4 \\
\hline & & \multirow[t]{2}{*}{ Pepe et al. (2002) } & \multirow[t]{2}{*}{$(\mathrm{B}-10)$} & \multirow[t]{2}{*}{2} & HD 108147 & 0.4 & 10.901 \\
\hline & & & & & HD 168746 & 0.23 & 6.403 \\
\hline & & \multirow[t]{4}{*}{ Udry et al.(2002) } & \multirow[t]{4}{*}{$(\mathrm{B}-11)$} & \multirow[t]{4}{*}{4} & HD 141937 & 9.7 & 653.22 \\
\hline & & & & & HD 162020 & 13.75 & 8.428198 \\
\hline & & & & & HD $168443 \mathrm{c}$ & 18.1 & 1765.8 \\
\hline & & & & & HD $202206 \mathrm{~b}$ & 17.4 & 255.87 \\
\hline & & Zucker et al. (2004) & $(\mathrm{B}-12)$ & 1 & HD $41004 \mathrm{~A}$ & 2.3 & 655 \\
\hline & & Santos et al. (2002) & $(\mathrm{B}-13)$ & 1 & HD $41004 \mathrm{~B}$ & 18.4 & 1.3283 \\
\hline & & Eggenberger et al.(2006) & $(\mathrm{B}-14)$ & 1 & HD 142022 & 4.4 & 1923 \\
\hline \multirow[t]{14}{*}{ (C) } & \multirow[t]{14}{*}{ Elodie } & Galland et al. (2005) & $(\mathrm{C}-1)$ & 1 & HD 33564 & 9.1 & 388 \\
\hline & & \multirow[t]{6}{*}{ Perrier et al. (2003) } & \multirow[t]{6}{*}{$(\mathrm{C}-2)$} & \multirow[t]{6}{*}{6} & HD 8574 & 2.23 & 228.8 \\
\hline & & & & & HD 23596 & 7.19 & 1558 \\
\hline & & & & & HD 33636 & 9.28 & 2127.7 \\
\hline & & & & & HD 50554 & 4.9 & 1279 \\
\hline & & & & & HD 106252 & 6.81 & 1500 \\
\hline & & & & & HD 190228 & 4.99 & 1127 \\
\hline & & \multirow[t]{3}{*}{ Naef et al.(2004) } & \multirow[t]{3}{*}{$(\mathrm{C}-3)$} & \multirow[t]{3}{*}{3} & HD $74156 \mathrm{~b}$ & 1.88 & 51.65 \\
\hline & & & & & HD $74156 \mathrm{c}$ & 8.03 & 2476 \\
\hline & & & & & HD $145675(14$ Her $)$ & 4.64 & 1773.4 \\
\hline & & Naef et al.(2003) & $(\mathrm{C}-4)$ & 1 & HD $190360 \mathrm{~b}$ & 1.502 & 2891 \\
\hline & & Moutou et al.(2006) & $(\mathrm{C}-5)$ & 1 & HD 185269 & 0.94 & 6.838 \\
\hline & & da. Silva et al.(2006) & $(\mathrm{C}-6)$ & 1 & HD 118203 & 2.13 & 6.1335 \\
\hline & & Mayor \& Queloz (1995) & $(\mathrm{C}-7)$ & 1 & $51 \mathrm{Peg}$ & 0.468 & 4.23077 \\
\hline (D) & HARPS & Udry et al. (2006) & $(\mathrm{D}-1)$ & 1 & HD 4308 & 0.047 & 15.56 \\
\hline
\end{tabular}




\begin{tabular}{|c|c|c|c|c|c|c|c|}
\hline Data Set & Survey & Reference & Ref. ID & $\mathrm{N}$ & Planet & $M$ & $P$ \\
\hline \multirow[t]{22}{*}{ (D) } & \multirow[t]{22}{*}{ HARPS } & \multirow[t]{3}{*}{ Moutou et al.(2005) } & \multirow[t]{3}{*}{$(\mathrm{D}-2)$} & \multirow[t]{3}{*}{3} & HD 2638 & 0.48 & 3.4442 \\
\hline & & & & & HD 27894 & 0.62 & 17.991 \\
\hline & & & & & HD 63454 & 0.38 & 2.81782 \\
\hline & & Pepe et al. (2004) & $(\mathrm{D}-3)$ & 1 & HD 330075 b & 0.76 & 3.369 \\
\hline & & Lo Curto et al.(2006) & $(\mathrm{D}-4)$ & 1 & HD 212301 & 0.45 & 2.457 \\
\hline & & \multirow[t]{3}{*}{ Lovis et al.(2005) } & \multirow[t]{3}{*}{$(\mathrm{D}-5)$} & \multirow[t]{3}{*}{3} & HD 93083 & 0.37 & 143.58 \\
\hline & & & & & HD 10193 & 0.3 & 70.46 \\
\hline & & & & & HD 102117 & 0.172 & 20.67 \\
\hline & & Santos et al.(2004) & $(\mathrm{D}-6)$ & 1 & HD $160691 \mathrm{~d}$ & 0.044 & 9.55 \\
\hline & & Pepe et al.(2007) & $(\mathrm{D}-7)$ & 1 & HD $160691 \mathrm{e}$ & 0.5219 & 310.55 \\
\hline & & Bonfils et al.(2005) & $(\mathrm{D}-8)$ & 1 & Gl $581 \mathrm{~b}$ & 0.0492 & 5.3683 \\
\hline & & \multirow[t]{2}{*}{ Udry et al.(2007) } & \multirow[t]{2}{*}{$(\mathrm{D}-9)$} & \multirow[t]{2}{*}{2} & Gl 581 c & 0.0158 & 12.932 \\
\hline & & & & & Gl $581 \mathrm{~d}$ & 0.0243 & 83.6 \\
\hline & & Bonfils et al.(2007) & $(\mathrm{D}-10)$ & 1 & GJ 674 & 0.037 & 4.6938 \\
\hline & & Melo et al.(2007) & $(\mathrm{D}-11)$ & 1 & HD 219828 & 0.066 & 3.8335 \\
\hline & & Santos et al. (2007) & $(\mathrm{D}-12)$ & 1 & HD 171028 & 1.83 & 538 \\
\hline & & \multirow[t]{3}{*}{ Naef et al. (2007) } & \multirow[t]{3}{*}{$(\mathrm{D}-13)$} & \multirow[t]{3}{*}{3} & HD 100777 & 1.16 & 383.7 \\
\hline & & & & & HD 190647 & 1.9 & 1038.1 \\
\hline & & & & & HD 221287 & 3.09 & 456.1 \\
\hline & & \multirow[t]{3}{*}{ Lovis et al. (2006) } & \multirow[t]{3}{*}{$(\mathrm{D}-14)$} & \multirow[t]{3}{*}{3} & HD $69830 \mathrm{~b}$ & 0.033 & 8.667 \\
\hline & & & & & HD $69830 \mathrm{c}$ & 0.038 & 31.56 \\
\hline & & & & & HD $69830 \mathrm{~d}$ & 0.058 & 197 \\
\hline \multirow[t]{10}{*}{$(\mathrm{E})$} & \multirow[t]{10}{*}{$\mathrm{N} 2 \mathrm{~K}$} & \multirow[t]{3}{*}{ Wright et al. (2007) } & \multirow[t]{3}{*}{$(\mathrm{E}-1)$} & \multirow[t]{3}{*}{3} & HIP 14810 b & 3.84 & 6.6742 \\
\hline & & & & & HIP $14810 \mathrm{c}$ & 0.76 & 95.2914 \\
\hline & & & & & HD 154345 b & 2.03 & 10900 \\
\hline & & \multirow[t]{3}{*}{ Johnson et al. (2006) } & \multirow[t]{3}{*}{$(\mathrm{E}-2)$} & \multirow[t]{3}{*}{3} & HD 33283 & 0.33 & 18.179 \\
\hline & & & & & HD 86081 & 1.5 & 2.1375 \\
\hline & & & & & HD 224693 & 0.71 & 26.73 \\
\hline & & \multirow[t]{2}{*}{ Fischer et al.(2006) } & \multirow[t]{2}{*}{$(\mathrm{E}-3)$} & \multirow[t]{2}{*}{2} & HD 149143 & 1.33 & 4.072 \\
\hline & & & & & HD 109749 & 0.28 & 5.24 \\
\hline & & \multirow[t]{2}{*}{ Fischer et al.(2007) } & $(\mathrm{E}-4)$ & 5 & HD 11506 & 4.85 & 1280 \\
\hline & & & & & & & \\
\hline
\end{tabular}




\begin{tabular}{|c|c|c|c|c|c|c|c|}
\hline Data Set & Survey & Reference & Ref. ID & $\mathrm{N}$ & Planet & $M$ & $P$ \\
\hline \multirow[t]{4}{*}{ (E) } & \multirow[t]{4}{*}{$\mathrm{N} 2 \mathrm{~K}$} & & & & HD 170469 & 0.67 & 1145 \\
\hline & & & & & HD $17156 \mathrm{~b}$ & 3.111 & 21.21725 \\
\hline & & Sato et al.(2005) & $(\mathrm{E}-5)$ & 1 & HD 149026 & 0.36 & 2.8766 \\
\hline & & Fischer et al.(2005) & $(\mathrm{E}-6)$ & 1 & HD 88133 & 0.22 & 3.41 \\
\hline \multirow[t]{28}{*}{$(\mathrm{F})$} & \multirow[t]{28}{*}{ Keck } & \multirow[t]{6}{*}{ Butler et al. (2003) } & \multirow[t]{6}{*}{$(\mathrm{F}-1)$} & \multirow[t]{6}{*}{6} & HD $108874 \mathrm{~b}$ & 1.36 & 395.4 \\
\hline & & & & & HD 114729 & 0.82 & 1131.478 \\
\hline & & & & & HD 72659 & 2.96 & 3177.4 \\
\hline & & & & & HD $128311 \mathrm{~b}$ & 2.18 & 448.6 \\
\hline & & & & & HD 49674 & 0.115 & 4.9437 \\
\hline & & & & & $\mathrm{HD} 37124 \mathrm{c}$ & 0.683 & 2295 \\
\hline & & \multirow[t]{5}{*}{ Marcy et al.(2005) } & \multirow[t]{5}{*}{$(\mathrm{F}-2)$} & \multirow[t]{5}{*}{5} & HD 183263 & 3.69 & 634.23 \\
\hline & & & & & HD 117207 & 2.06 & 2627.08 \\
\hline & & & & & HD 188015 & 1.26 & 456.46 \\
\hline & & & & & HD 45350 & 1.79 & 890.76 \\
\hline & & & & & HD 99492 & 0.109 & 17.0431 \\
\hline & & \multirow[t]{2}{*}{ Robinson et al.(2007) } & \multirow[t]{2}{*}{$(\mathrm{F}-3)$} & \multirow[t]{2}{*}{2} & HD 75898 b & 1.48 & 204.2 \\
\hline & & & & & HD 5319 b & 1.94 & 675 \\
\hline & & Butler et al.(1998) & $(\mathrm{F}-4)$ & 1 & HD $187123 \mathrm{~b}$ & 0.52 & 3.097 \\
\hline & & \multirow[t]{2}{*}{ Marcy et al.(1999) } & \multirow[t]{2}{*}{$(\mathrm{F}-5)$} & \multirow[t]{2}{*}{2} & HD 210277 & 1.23 & 442.1 \\
\hline & & & & & HD $168443 \mathrm{~b}$ & 8.02 & 58.11289 \\
\hline & & Johnson et al.(2007) & $(\mathrm{F}-6)$ & 1 & GJ 317 & 1.2 & 692.9 \\
\hline & & \multirow[t]{6}{*}{ Vogt et al.(2000) } & \multirow[t]{6}{*}{$(\mathrm{F}-7)$} & \multirow[t]{6}{*}{6} & HD 10697 & 6.12 & 1077.906 \\
\hline & & & & & HD $37124 \mathrm{~b}$ & 0.61 & 154.46 \\
\hline & & & & & HD 134987 & 1.58 & 260 \\
\hline & & & & & HD 177830 & 1.28 & 391 \\
\hline & & & & & HD 192263 & 0.72 & 24.348 \\
\hline & & & & & HD 22258 & 5.11 & 572 \\
\hline & & Butler et al.(2006b) & $(\mathrm{F}-8)$ & 1 & GJ 849 & 0.82 & 1890 \\
\hline & & \multirow[t]{4}{*}{ Vogt et al. (2002) } & \multirow[t]{4}{*}{$(\mathrm{F}-9)$} & \multirow[t]{4}{*}{5} & HD 4203 & 1.65 & 400.944 \\
\hline & & & & & HD 4208 & 0.8 & 812.197 \\
\hline & & & & & HD 33636 & 9.28 & 2127.7 \\
\hline & & & & & & & \\
\hline
\end{tabular}




\begin{tabular}{|c|c|c|c|c|c|c|c|}
\hline Data Set & Survey & Reference & Ref. ID & $\mathrm{N}$ & Planet & $M$ & $P$ \\
\hline \multirow{19}{*}{$(\mathrm{F})$} & \multirow[t]{19}{*}{ Keck } & Marcy et al.(2001a) & $(\mathrm{F}-10)$ & 1 & HD $168443 \mathrm{c}$ & 18.1 & 1765.8 \\
\hline & & Rivera et al. (2005) & $(\mathrm{F}-11)$ & 1 & GJ $876 \mathrm{~d}$ & 0.018 & 1.93776 \\
\hline & & \multirow[t]{7}{*}{ Vogt et al.(2005) } & \multirow[t]{7}{*}{$(\mathrm{F}-12)$} & \multirow[t]{7}{*}{7} & HD $128311 \mathrm{c}$ & 3.21 & 919 \\
\hline & & & & & HD 50449 & 1.71 & 2582.7 \\
\hline & & & & & HD $37124 \mathrm{~d}$ & 0.6 & 843.6 \\
\hline & & & & & HD $190360 \mathrm{c}$ & 0.057 & 17.1 \\
\hline & & & & & HD $108874 \mathrm{c}$ & 1.018 & 1605.8 \\
\hline & & & & & HD $37124 \mathrm{c}$ & 0.683 & 2295 \\
\hline & & & & & HD $217107 \mathrm{c}$ & 2.5 & 3352 \\
\hline & & Butler et al.(2004) & $(\mathrm{F}-13)$ & 1 & GJ 436 & 0.072 & 2.64385 \\
\hline & & \multirow[t]{5}{*}{ Butler et al.(2006a) } & \multirow[t]{5}{*}{$(\mathrm{F}-14)$} & \multirow[t]{5}{*}{5} & HD 11964 b & 0.11 & 37.82 \\
\hline & & & & & HD 66428 & 2.82 & 1973 \\
\hline & & & & & HD 99109 & 0.502 & 439.3 \\
\hline & & & & & HD 107148 & 0.21 & 48.056 \\
\hline & & & & & HD 164922 & 0.36 & 1155 \\
\hline & & \multirow[t]{2}{*}{ Marcy et al.(2000) } & \multirow[t]{2}{*}{$(\mathrm{F}-15)$} & \multirow[t]{2}{*}{2} & HD 16141 & 0.23 & 75.56 \\
\hline & & & & & HD 46375 & 0.249 & 3.024 \\
\hline & & \multirow[t]{2}{*}{ Butler et al.(2000) } & \multirow[t]{2}{*}{$(\mathrm{F}-16)$} & \multirow[t]{2}{*}{2} & HD 52265 & 1.13 & 118.96 \\
\hline & & & & & BD 103166 & 0.48 & 3.488 \\
\hline \multirow[t]{12}{*}{$(\mathrm{G})$} & \multirow[t]{12}{*}{ AAPS } & \multirow[t]{4}{*}{ Tinney et al.(2003) } & \multirow[t]{4}{*}{$(\mathrm{G}-1)$} & \multirow[t]{4}{*}{4} & HD 73526 b & 2.9 & 188.3 \\
\hline & & & & & HD 76700 & 0.197 & 3.971 \\
\hline & & & & & HD 30177 & 9.17 & 2819.654 \\
\hline & & & & & HD 2039 & 4.85 & 1192.582 \\
\hline & & \multirow[t]{3}{*}{ Jones et al.(2002b) } & \multirow[t]{3}{*}{$(\mathrm{G}-2)$} & \multirow[t]{3}{*}{3} & HD 196050 & 3 & 1289 \\
\hline & & & & & HD 216437 & 2.1 & 1294 \\
\hline & & & & & HD $160691 \mathrm{c}$ & 3.1 & 2986 \\
\hline & & Jones et al.(2002a) & $(\mathrm{G}-3)$ & 1 & HD 39091 & 10.35 & 2063.818 \\
\hline & & \multirow[t]{2}{*}{ Tinney et al.(2002) } & \multirow[t]{2}{*}{$(\mathrm{G}-4)$} & \multirow[t]{2}{*}{2} & HD 142 & 1 & 337.112 \\
\hline & & & & & HD 23079 & 2.61 & 738.459 \\
\hline & & \multirow[t]{2}{*}{ Jones et al.(2006) } & \multirow[t]{2}{*}{$(\mathrm{G}-5)$} & \multirow[t]{2}{*}{2} & HD 187085 & 0.75 & 986 \\
\hline & & & & & HD 20782 & 1.8 & 585.86 \\
\hline
\end{tabular}




\begin{tabular}{|c|c|c|c|c|c|c|c|}
\hline Data Set & Survey & Reference & Ref. ID & $\mathrm{N}$ & Planet & $M$ & $P$ \\
\hline \multirow[t]{11}{*}{$(G)$} & \multirow[t]{11}{*}{ AAPS } & \multirow[t]{2}{*}{ O'Toole et al.(2007) } & \multirow[t]{2}{*}{$(\mathrm{G}-6)$} & \multirow[t]{2}{*}{2} & HD 23127 & 1.5 & 1214 \\
\hline & & & & & HD 159868 & 1.7 & 986 \\
\hline & & \multirow[t]{2}{*}{ Butler et al.(2001) } & \multirow[t]{2}{*}{$(\mathrm{G}-7)$} & \multirow[t]{2}{*}{2} & HD 160691 b & 1.67 & 654.5 \\
\hline & & & & & HD 27442 & 1.28 & 423.841 \\
\hline & & Carter et al.(2003) & $(\mathrm{G}-8)$ & 1 & HD 70642 & 2 & 2231 \\
\hline & & Tinney et al.(2001) & $(\mathrm{G}-9)$ & 1 & HD 179949 & 0.95 & 3.0925 \\
\hline & & Jones et al.(2003) & $(\mathrm{G}-10)$ & 1 & Tau Gruis b & 1.49 & 1442.919 \\
\hline & & Tinney et al.(2006) & $(\mathrm{G}-11)$ & 1 & HD73526 c & 1.6 & 416.1 \\
\hline & & \multirow[t]{3}{*}{ Tinney et al.(2005) } & \multirow[t]{3}{*}{$(\mathrm{G}-12)$} & \multirow[t]{3}{*}{3} & HD 117618 & 0.19 & 52.2 \\
\hline & & & & & HD 208487 & 0.45 & 123 \\
\hline & & & & & HD 102117 & 0.172 & 20.67 \\
\hline \multirow[t]{17}{*}{$(\mathrm{H})$} & \multirow[t]{17}{*}{ Others } & \multirow[t]{3}{*}{ Fischer et al. (2001) } & \multirow[t]{3}{*}{$(\mathrm{H}-1)$} & \multirow[t]{3}{*}{3} & HD $12661 \mathrm{~b}$ & 2.3 & 263.6 \\
\hline & & & & & HD 92788 & 3.86 & 377.7 \\
\hline & & & & & HD $38529 \mathrm{~b}$ & 0.78 & 14.30 \\
\hline & & Marcy et al.(2001b) & $(\mathrm{H}-2)$ & 1 & GJ $876 \mathrm{c}$ & 0.56 & 30.1 \\
\hline & & \multirow[t]{3}{*}{ Fischer et al. (2003) } & \multirow[t]{3}{*}{$(\mathrm{H}-3)$} & \multirow[t]{3}{*}{3} & HD 40979 & 3.32 & 267.2 \\
\hline & & & & & HD $12661 \mathrm{c}$ & 1.57 & 1444.5 \\
\hline & & & & & HD 38529 c & 12.7 & 2174.3 \\
\hline & & Delfosse et al.(1998) & $(\mathrm{H}-4)$ & 1 & GJ 876 b & 1.935 & 60.94 \\
\hline & & Cochran et al. (1997) & $(\mathrm{H}-5)$ & 1 & 16 Cyg B b & 1.68 & 799.5 \\
\hline & & Sozzetti et al. (2006) & $(\mathrm{H}-6)$ & 1 & HD 81040 & 6.86 & 1001.7 \\
\hline & & Naef et al.(2001b) & $(\mathrm{H}-7)$ & 1 & HD $80606 \mathrm{~b}$ & 3.41 & 111.78 \\
\hline & & \multirow[t]{2}{*}{ Fischer et al. (1999) } & \multirow[t]{2}{*}{$(\mathrm{H}-8)$} & \multirow[t]{2}{*}{2} & HD 195019 A b & 3.7 & 18.20163 \\
\hline & & & & & HD 217107 & 1.33 & 7.12689 \\
\hline & & \multirow[t]{2}{*}{ Butler et al.(1999) } & \multirow[t]{2}{*}{$(\mathrm{H}-9)$} & \multirow[t]{2}{*}{2} & Ups And c & 1.98 & 241.52 \\
\hline & & & & & Ups And d et al. & 3.95 & 1274.6 \\
\hline & & Fischer et al.(2002b) & $(\mathrm{H}-10)$ & 1 & HD 136118 & 11.9 & 1209 \\
\hline & & Korzennik et al.(2000) & $(\mathrm{H}-11)$ & 1 & HD 89744 & 7.99 & 256.605 \\
\hline
\end{tabular}




\section{Appendix B}

The derivation of the $\log$ likelihood $\ln L$ of coupled mass-period functions is shown here. As in Tabachnik and Tremaine (2002), the likelihood function is

$$
\begin{aligned}
L & =\prod_{i=1}^{N} n\left(x_{i}, y_{i}\right) \exp \left[-\int_{D} n(x, y) d x d y\right] \\
& =\prod_{i=1}^{N} c N_{s} f_{X Y}\left(x_{i}, y_{i}\right) \exp \left[-c N_{s} \int_{y_{\min }}^{\tilde{u}} \int_{v+y / 3}^{x_{\max }} f_{X Y}(x, y) d x d y\right] .
\end{aligned}
$$

By the other equations in $\S 6$, we have

$$
\begin{aligned}
& \int_{y_{\min }}^{\tilde{u}} \int_{v+y / 3}^{x_{\max }} f_{X Y}(x, y) d x d y=\int_{y_{\min }}^{\tilde{u}} \int_{v+y / 3}^{x_{\max }} \frac{\partial^{2} C\left(F_{X}(x), F_{Y}(y) ; \theta\right)}{\partial F_{X} \partial F_{Y}} f_{X}(x) f_{Y}(y) d x d y, \\
= & \int_{y_{\min }}^{\tilde{u}}\left\{\frac{\partial C\left(F_{X}\left(x_{\max }\right), F_{Y}(y) ; \theta\right)}{\partial F_{Y}}-\frac{\partial C\left(F_{X}(v+y / 3), F_{Y}(y) ; \theta\right)}{\partial F_{Y}}\right\} f_{Y}(y) d y, \\
= & C\left(F_{X}\left(x_{\max }\right), F_{Y}(\tilde{u}) ; \theta\right)-C\left(F_{X}\left(x_{\max }\right), F_{Y}\left(y_{\min }\right) ; \theta\right)-\int_{y_{\min }}^{\tilde{u}} \frac{\partial C\left(F_{X}(v+y / 3), F_{Y}(y) ; \theta\right)}{\partial F_{Y}} f_{Y}(y) d y, \\
= & F_{Y}(\tilde{u})-\int_{y_{\min }}^{\tilde{u}} \frac{\left[e^{-\theta\left(F_{Y}(y)+F_{X}(v+y / 3)\right)}-e^{-\theta F_{Y}(y)}\right]}{e^{-\theta}+e^{-\theta\left(F_{Y}(y)+F_{X}(v+y / 3)\right)}-e^{-\theta F_{Y}(y)}-e^{-\theta F_{X}(v+y / 3)}} f_{Y}(y) d y,
\end{aligned}
$$

where $C\left(F_{X}\left(x_{\max }\right), F_{Y}\left(y_{\min }\right) ; \theta\right)=C(1,0, \theta)=0$, and $C\left(F_{X}\left(x_{\max }\right), F_{Y}(\tilde{u}) ; \theta\right)=C\left(1, F_{Y}(\tilde{u}) ; \theta\right)=$ $F_{Y}(\tilde{u})$. From Eq.(37) and Eq.(38), we have

$$
\begin{aligned}
& \exp \left[-\int_{D} n(x, y) d x d y\right] \\
= & \exp \left\{-c N_{s}\left(F_{Y}(\tilde{u})-\int_{y_{\min }}^{\tilde{u}} \frac{\left[e^{-\theta\left(F_{Y}(y)+F_{X}(v+y / 3)\right)}-e^{-\theta F_{Y}(y)}\right] f_{Y}(y)}{e^{-\theta}+e^{-\theta\left(F_{Y}(y)+F_{X}(v+y / 3)\right)}-e^{-\theta F_{Y}(y)}-e^{-\theta F_{X}(v+y / 3)}} d y\right)\right\},
\end{aligned}
$$

and the log-likelihood is

$$
\begin{aligned}
\ln L= & N \ln \left(c N_{s}\right)-\alpha \sum_{i=1}^{N} x_{i}-\beta \sum_{i=1}^{N} y_{i}+N \ln \left(\frac{\alpha}{e^{-\alpha x_{\min }}-e^{-\alpha x_{\max }}}\right)+N \ln \left(\frac{\beta}{e^{-\beta y_{\min }}-e^{-\beta y_{\max }}}\right) \\
& +\sum_{i=1}^{N} \ln \left(C_{u_{1} u_{2}}\left(F_{X}\left(x_{i}\right), F_{Y}\left(y_{i}\right) ; \theta\right)\right)-c N_{s} I(\alpha, \beta, u, v)
\end{aligned}
$$

where

$$
\begin{gathered}
C_{u_{1} u_{2}}\left(F_{X}\left(x_{i}\right), F_{Y}\left(y_{i}\right) ; \theta\right)=\frac{-\theta\left(e^{-\theta}-1\right) e^{-\theta F_{X}\left(x_{i}\right)} e^{-\theta F_{Y}\left(y_{i}\right)}}{\left[e^{-\theta}-1+\left(e^{-\theta F_{X}\left(x_{i}\right)}-1\right)\left(e^{-\theta F_{Y}\left(y_{i}\right)}-1\right)\right]^{2}} \\
I(\alpha, \beta, u, v)=F_{Y}(\tilde{u})-\int_{y_{\min }}^{\tilde{u}} \frac{\left[e^{-\theta\left(F_{Y}(y)+F_{X}(v+y / 3)\right)}-e^{-\theta F_{Y}(y)}\right] f_{Y}(y)}{e^{-\theta}+e^{-\theta\left(F_{Y}(y)+F_{X}(v+y / 3)\right)}-e^{-\theta F_{Y}(y)}-e^{\left.-\theta F_{X}(v+y / 3)\right)}} d y .
\end{gathered}
$$


By $\partial \ln L / \partial c=0$, we obtain $c=N /\left[N_{s} I(\alpha, \beta, u, v)\right]$. It is then substituted into Eq.(40) and we have

$$
\begin{aligned}
\ln L= & N \ln (N)-N \ln (I)-\alpha \sum_{i=1}^{N} x_{i}-\beta \sum_{i=1}^{N} y_{i}+N \ln \left(\frac{\alpha}{e^{-\alpha x_{\min }}-e^{-\alpha x_{\max }}}\right) \\
& +N \ln \left(\frac{\beta}{e^{-\beta y_{\min }}-e^{-\beta y_{\max }}}\right)+\sum_{i=1}^{N} \ln \left(C_{u_{1} u_{2}}\left(F_{X}\left(x_{i}\right), F_{Y}\left(y_{i}\right) ; \theta\right)\right)-N .
\end{aligned}
$$




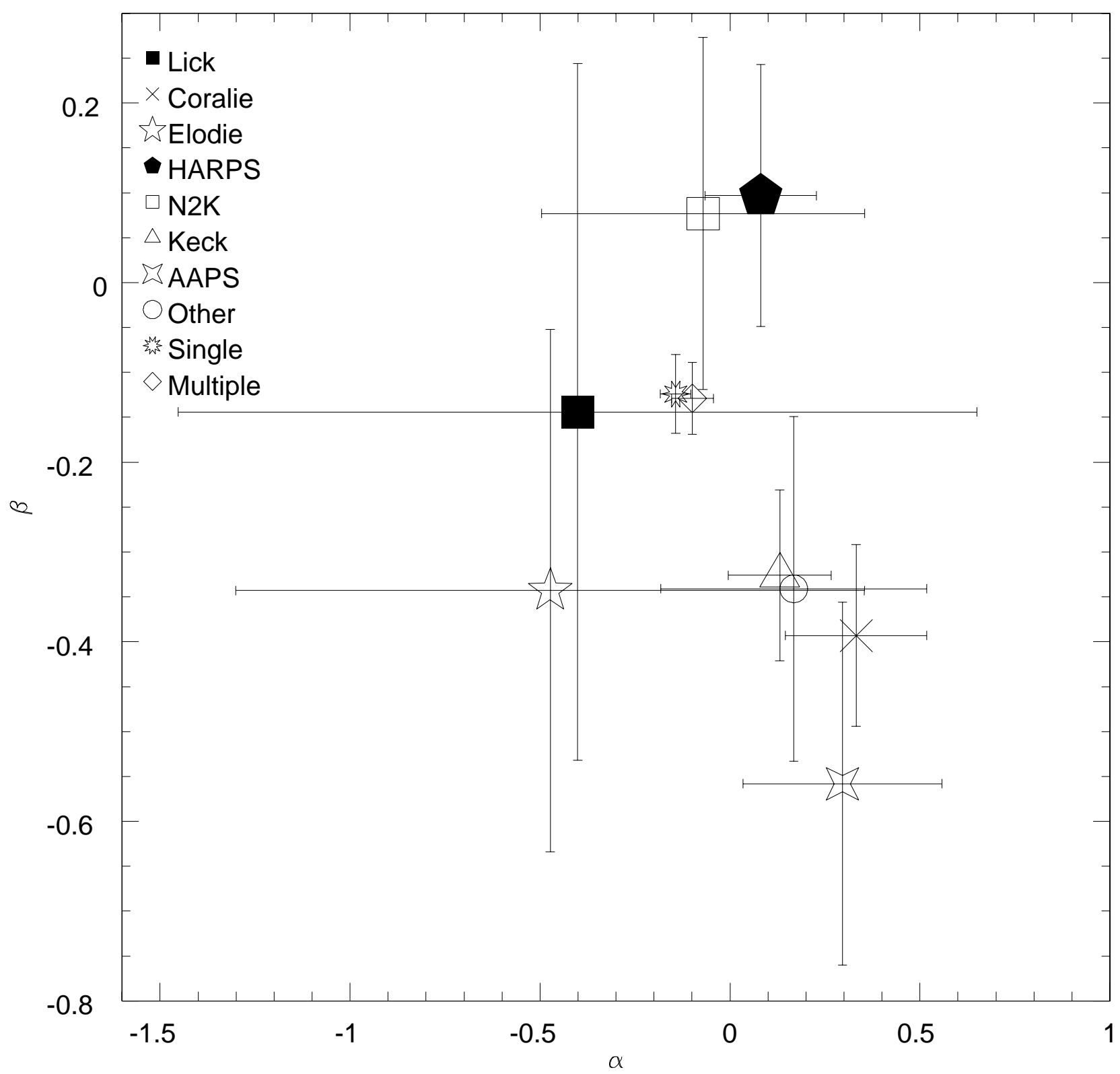

Fig. 1. - The estimators of the exponents, $\alpha$ and $\beta$, with error bars for all surveys listed in Table 1 , and also the results of Multiple Surveys. 


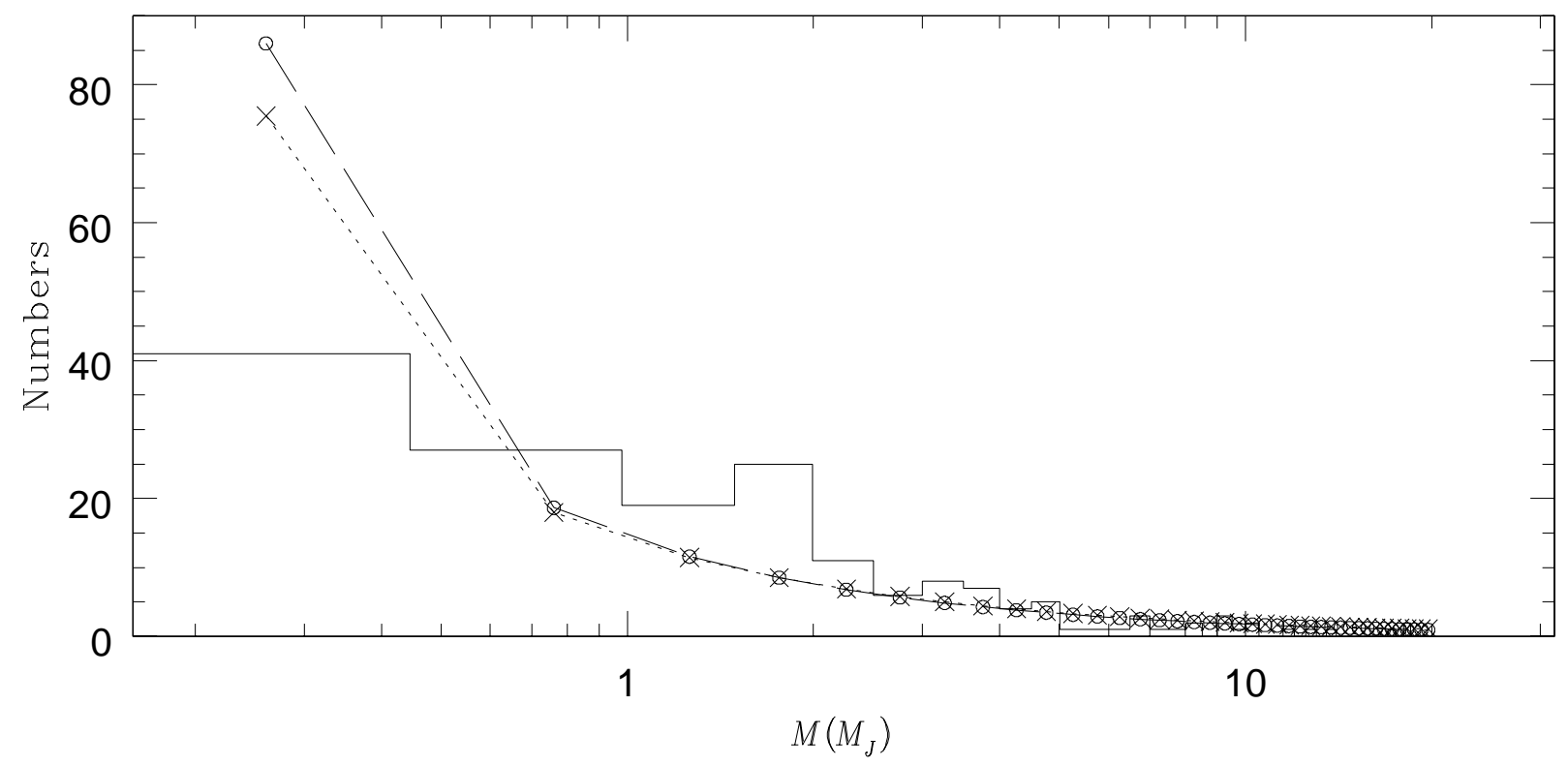

(a)

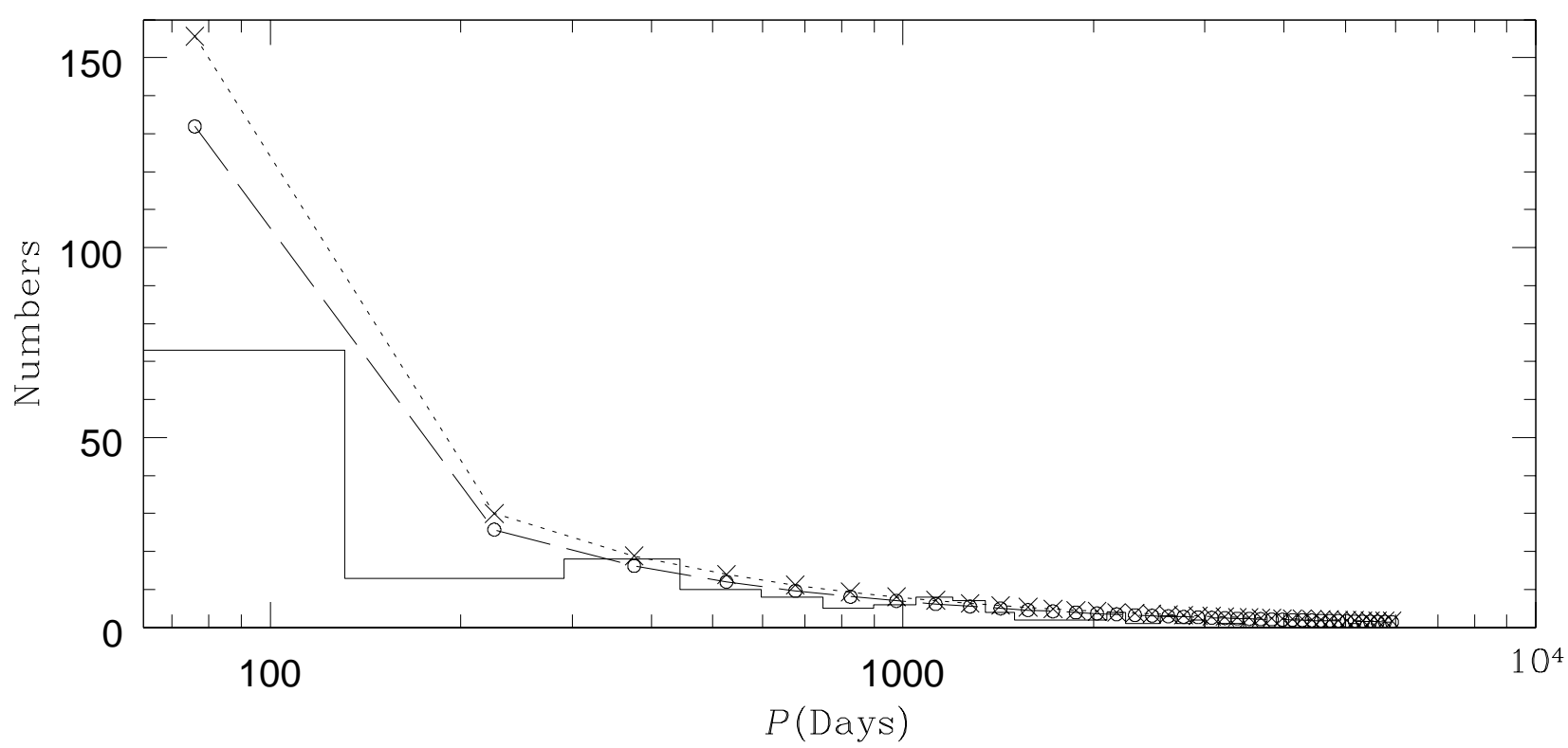

(b)

Fig. 2.- The distributions of exoplanets in $M$ and $P$ space. The solid lines are the histograms of 175 exoplanets. By Eq.(24), the crosses with dotted curves are the results of Single Imaginary Survey, and the circles with dashed curves are the results of Multiple Surveys. 


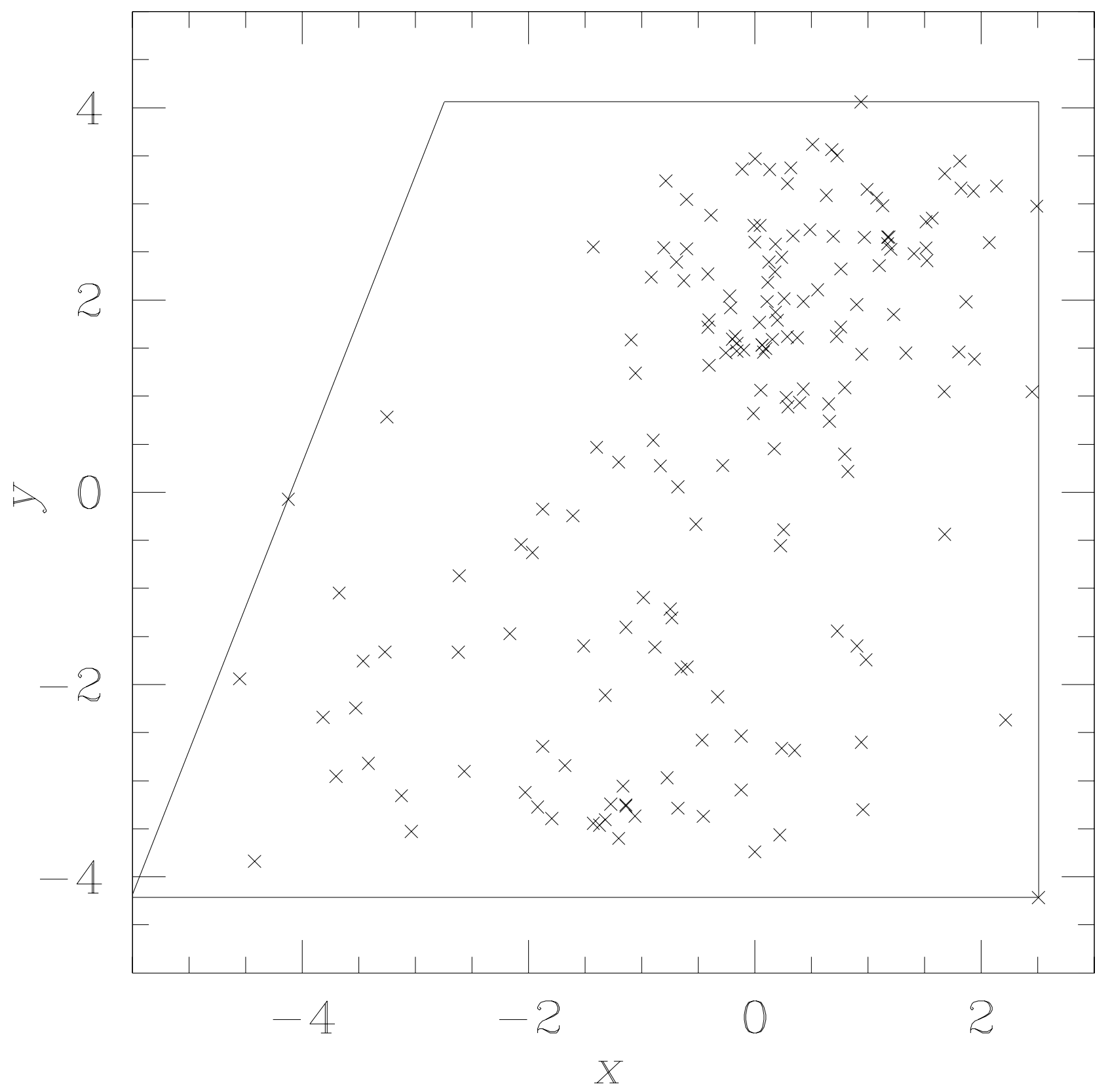

Fig. 3.- The distribution of 175 exoplanets in $x-y$ space. The solid lines indicate Domain D. 


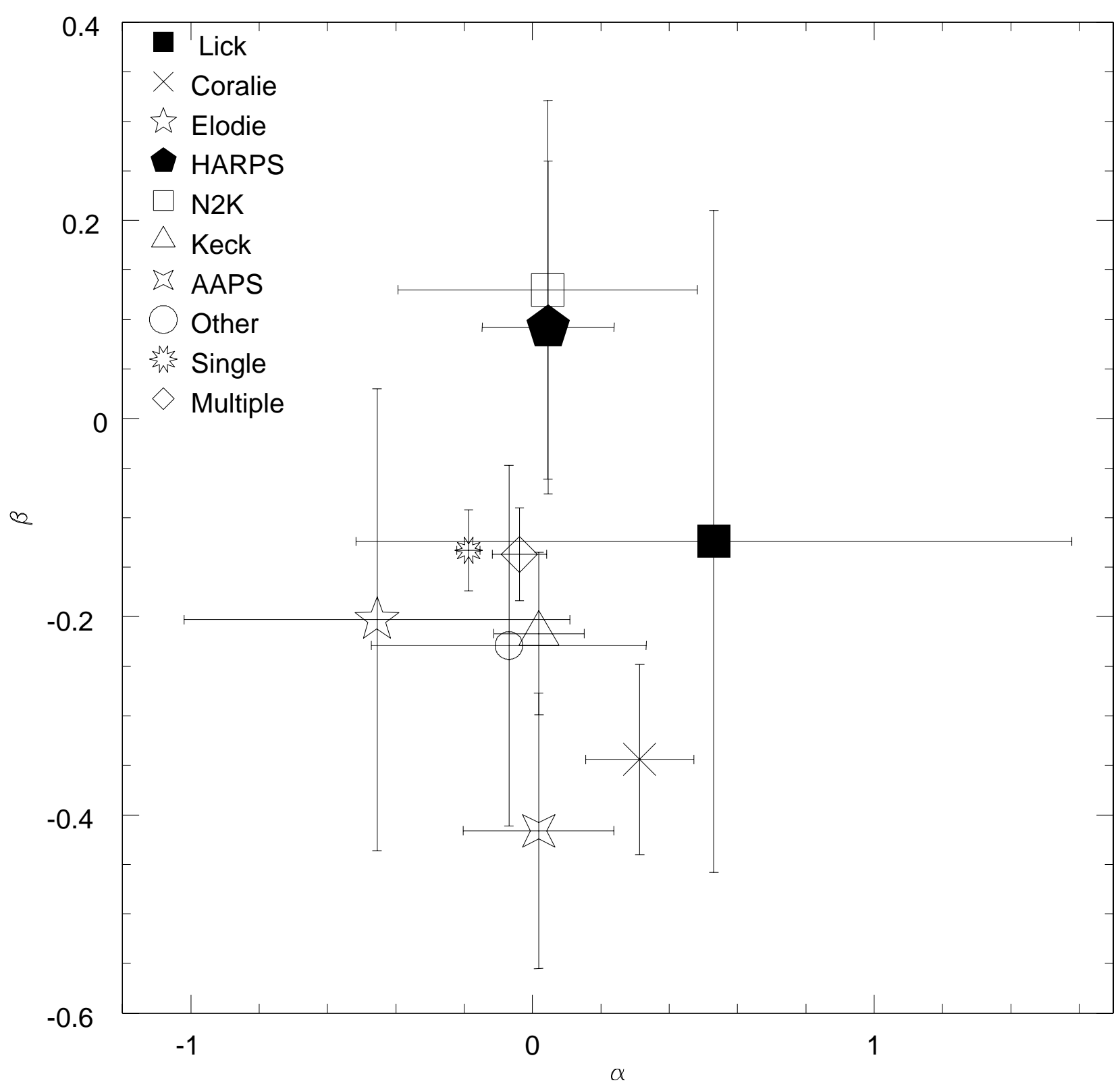

Fig. 4.- The estimators of the exponents, $\alpha$ and $\beta$, with error bars for all surveys listed in Table 4 . 
(a)

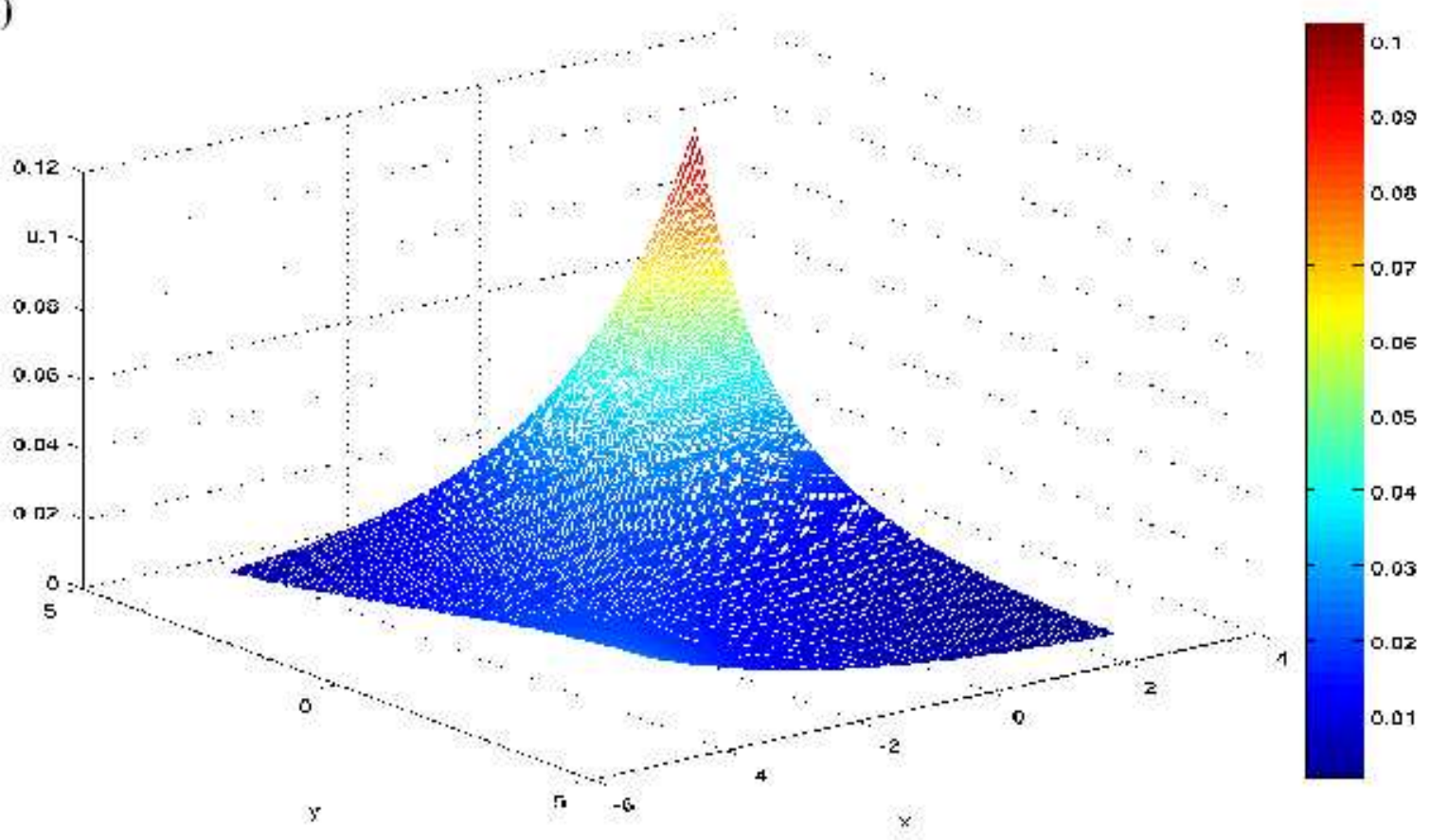

(b)

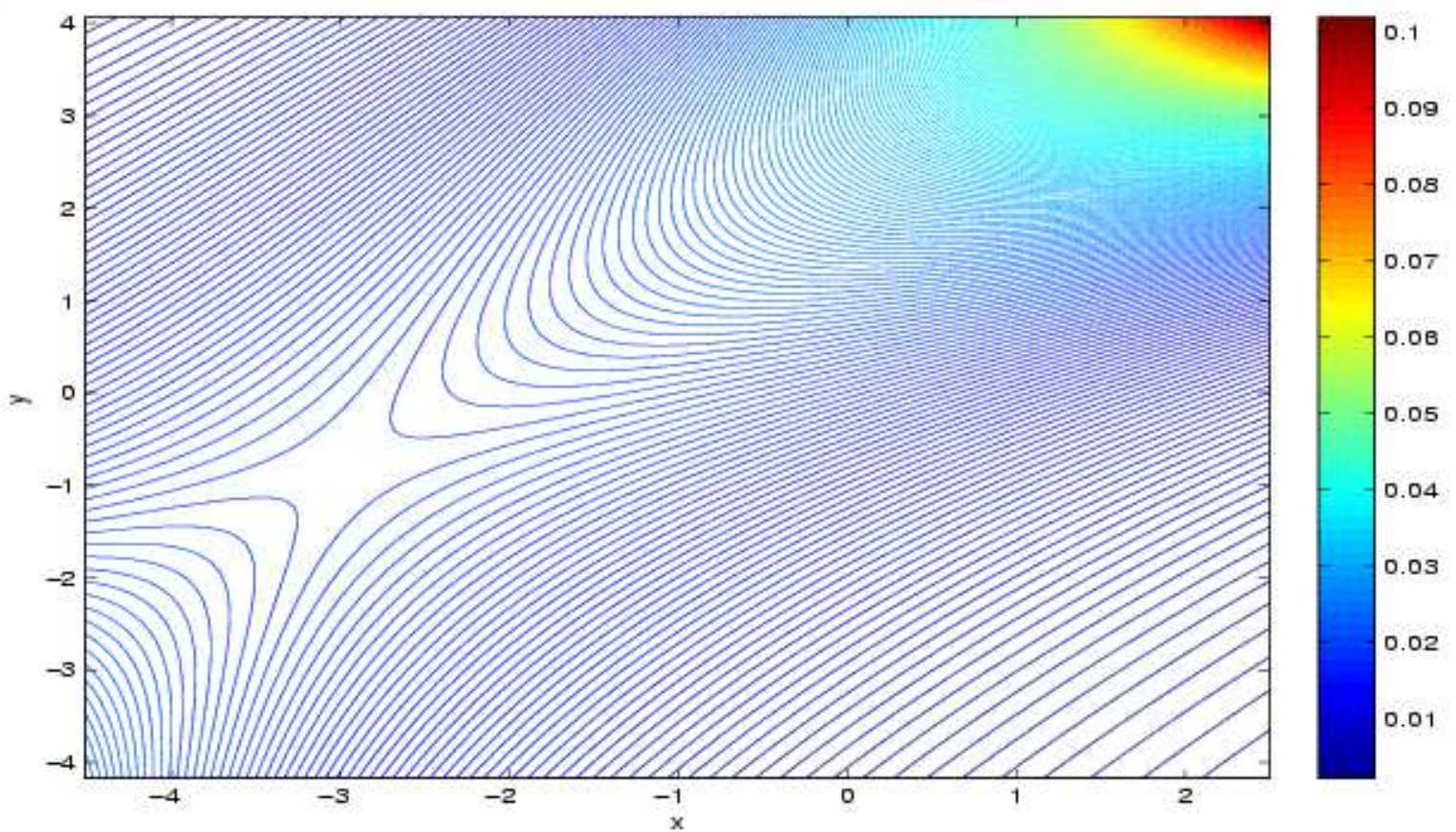

Fig. 5.- (a) The coupled probability density function in $x-y$ space, i.e. $f_{X Y}(x, y)$, from the results of Multiple Surveys. (b) The corresponding contour plot. 


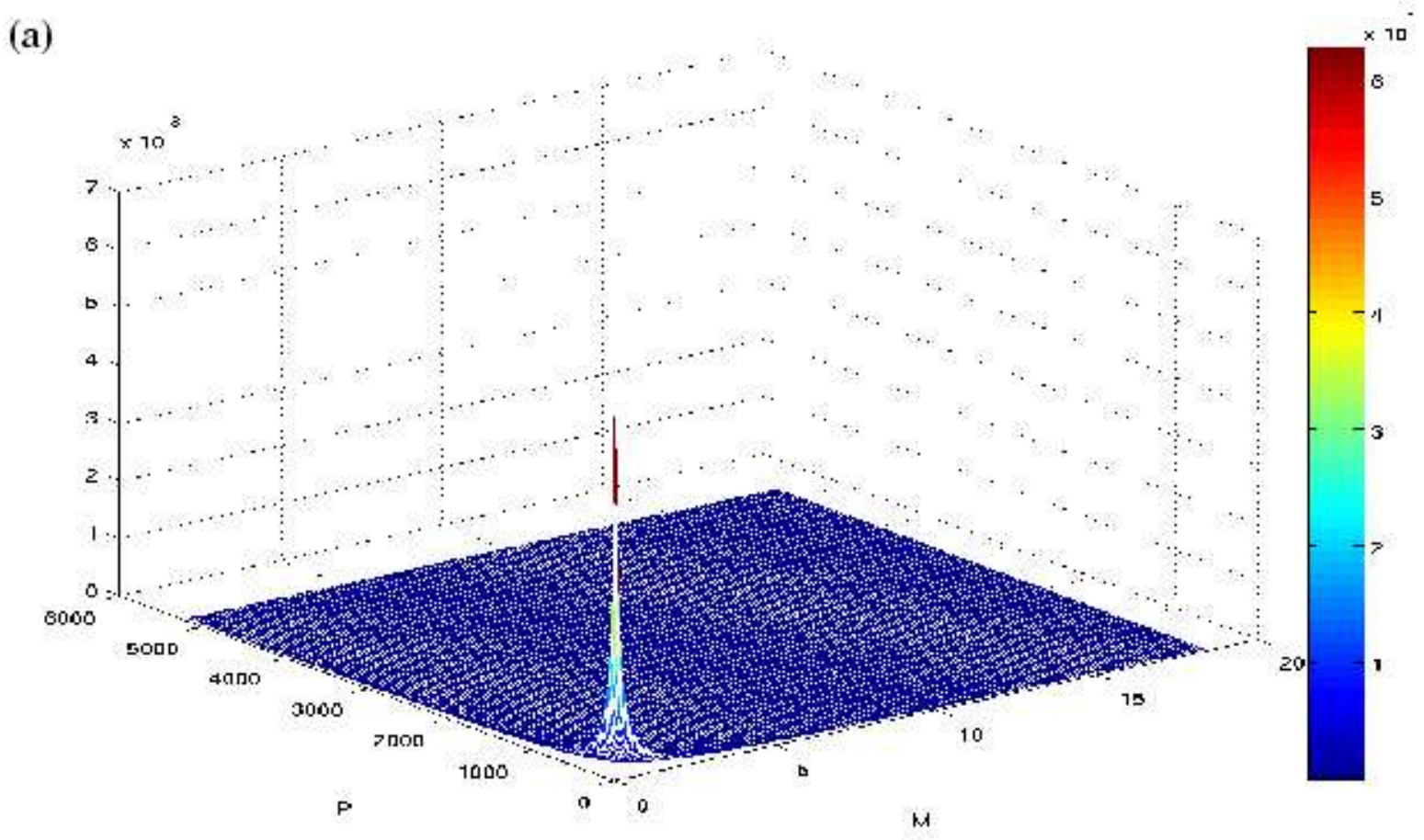

(b)

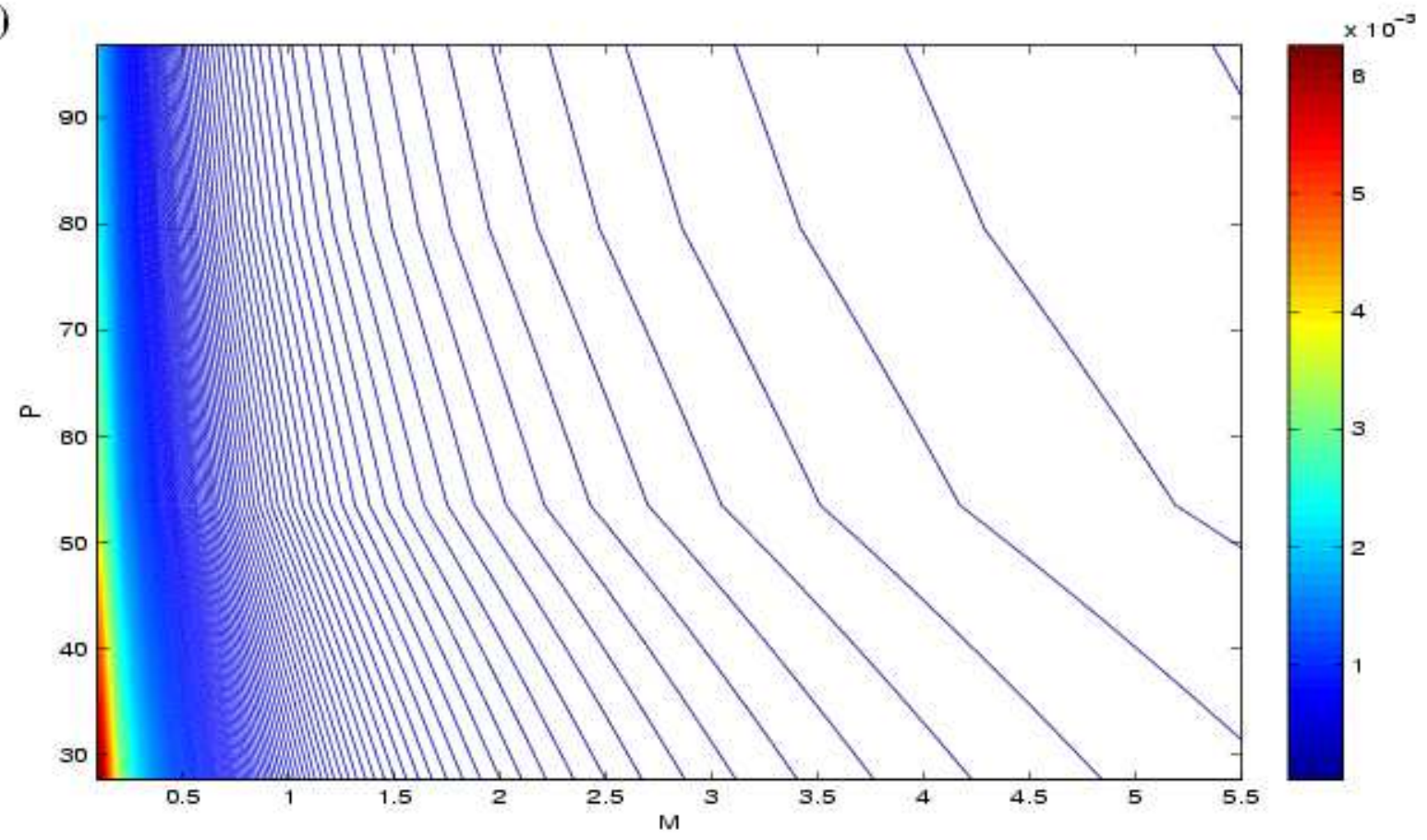

Fig. 6. - (a) The coupled probability density function in $M-P$ space, i.e. $f_{M P}(M, P)$, from the results of Multiple Surveys. (b) The corresponding contour plot. 
(a)

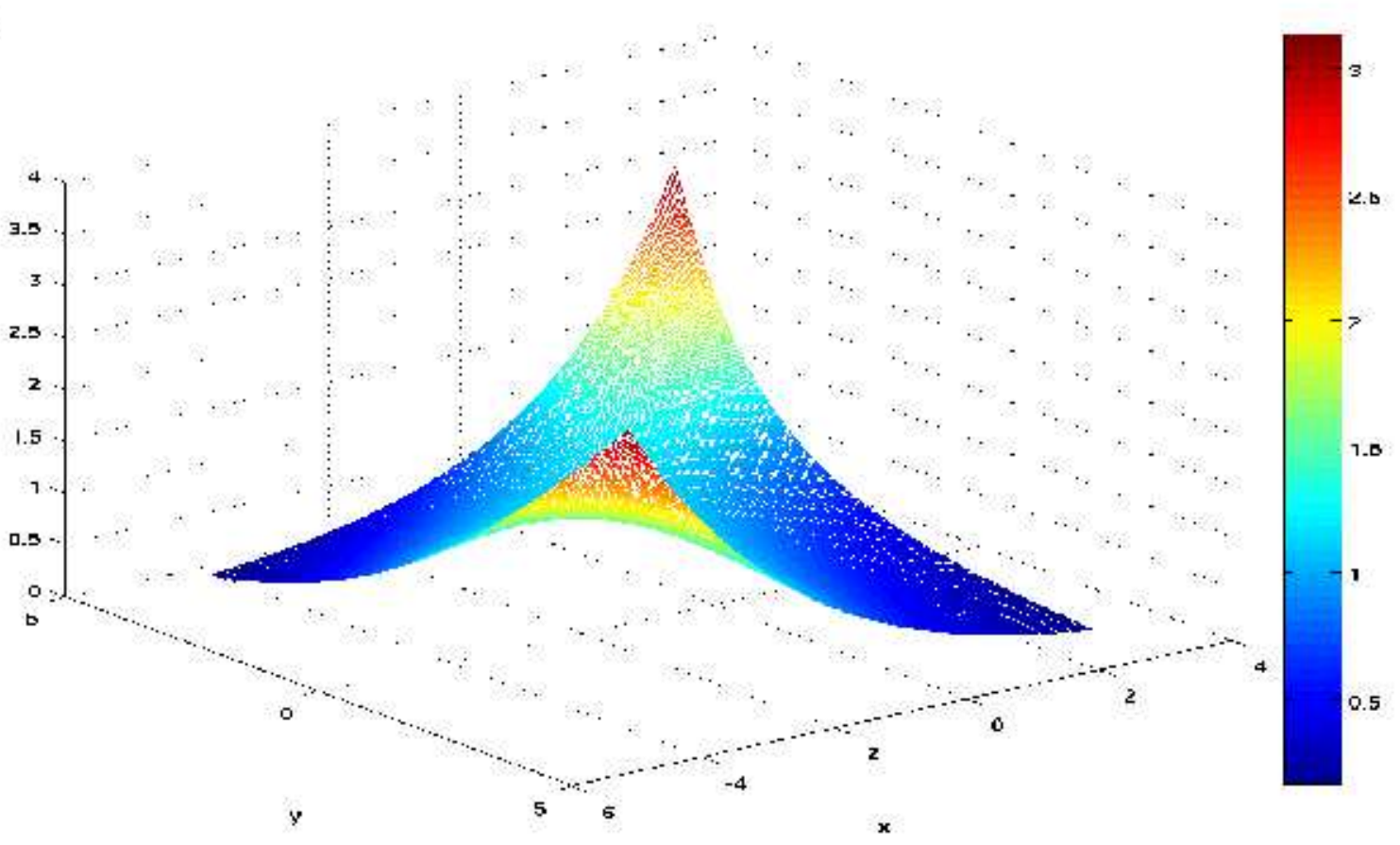

(b)

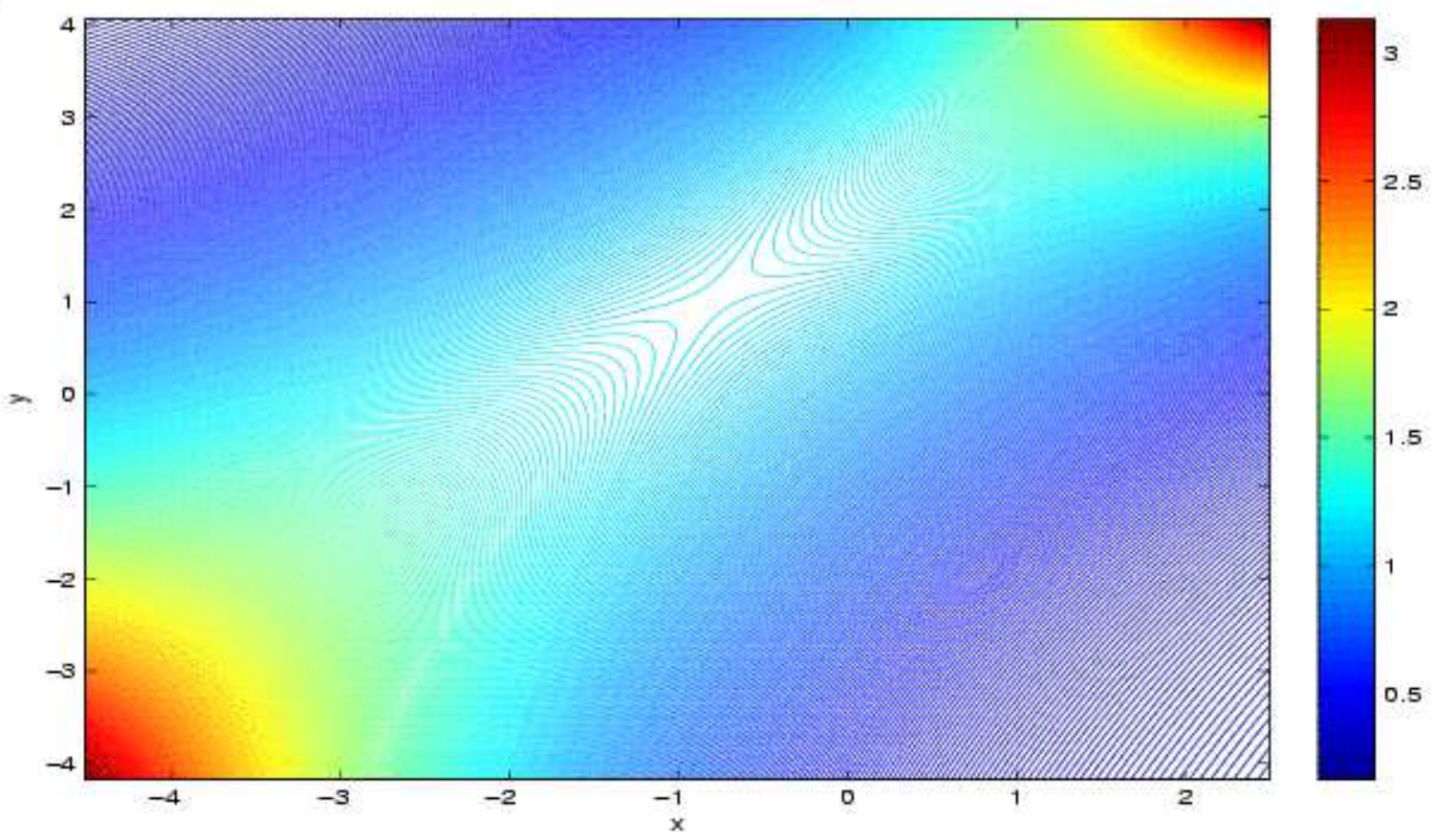

Fig. 7.- (a) The three-dimensional plot of the coupling factor for the results of Multiple Surveys. (b) The corresponding contour plot. 

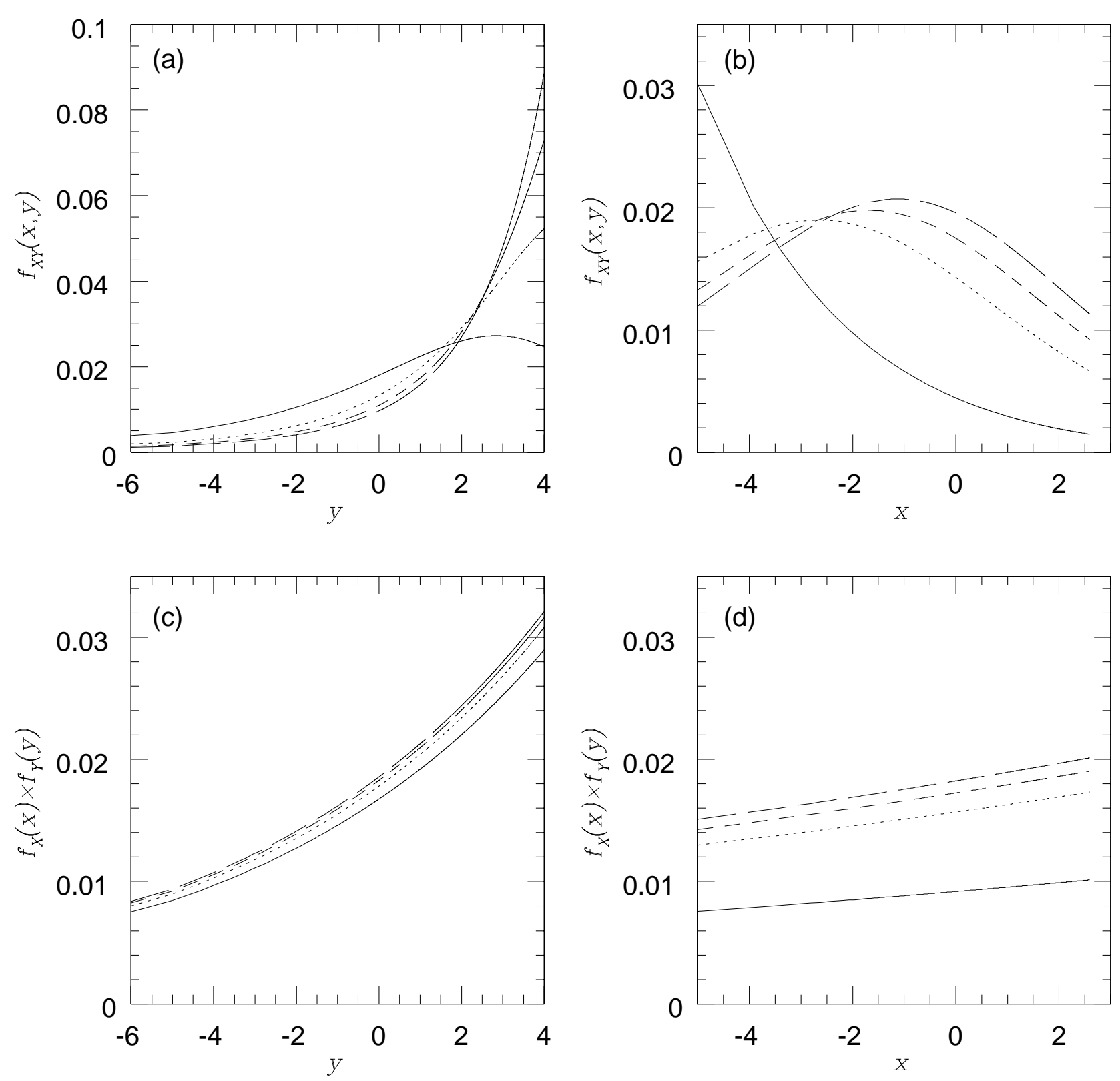

Fig. 8. - The mass and period functions in $x-y$ space for the results of Multiple Surveys. (a) The period functions of $x=\ln \left(1 M_{J} / M_{0}\right)$ (solid curve), $x=\ln \left(5 M_{J} / M_{0}\right)$ (dotted curve), $x=\ln \left(10 M_{J} / M_{0}\right)$ (short dashed curve), and $x=\ln \left(15 M_{J} / M_{0}\right)$ (long dashed curve). (b) The mass functions of $y=\ln \left(1\right.$ day $\left./ P_{0}\right)$ (solid curve), $y=\ln \left(50\right.$ days $\left./ P_{0}\right)$ (dotted curve), $y=\ln \left(100\right.$ days $\left./ P_{0}\right)$ (short dashed curve), and $y=\ln \left(150\right.$ days $\left./ P_{0}\right)$ (long dashed curve). (c) The independent period functions of $x=\ln \left(1 M_{J} / M_{0}\right)$ (solid curve), $x=\ln \left(5 M_{J} / M_{0}\right) \quad$ (dotted curve), $x=\ln \left(10 M_{J} / M_{0}\right)$ (short dashed curve), and $x=\ln \left(15 M_{J} / M_{0}\right)$ (long dashed curve). (d) The independent mass functions of $y=\ln \left(1\right.$ day $\left./ P_{0}\right)$ (solid curve), $y=\ln \left(50\right.$ days $\left./ P_{0}\right)$ (dotted curve), $y=\ln \left(100\right.$ days $\left./ P_{0}\right)$ (short dashed 

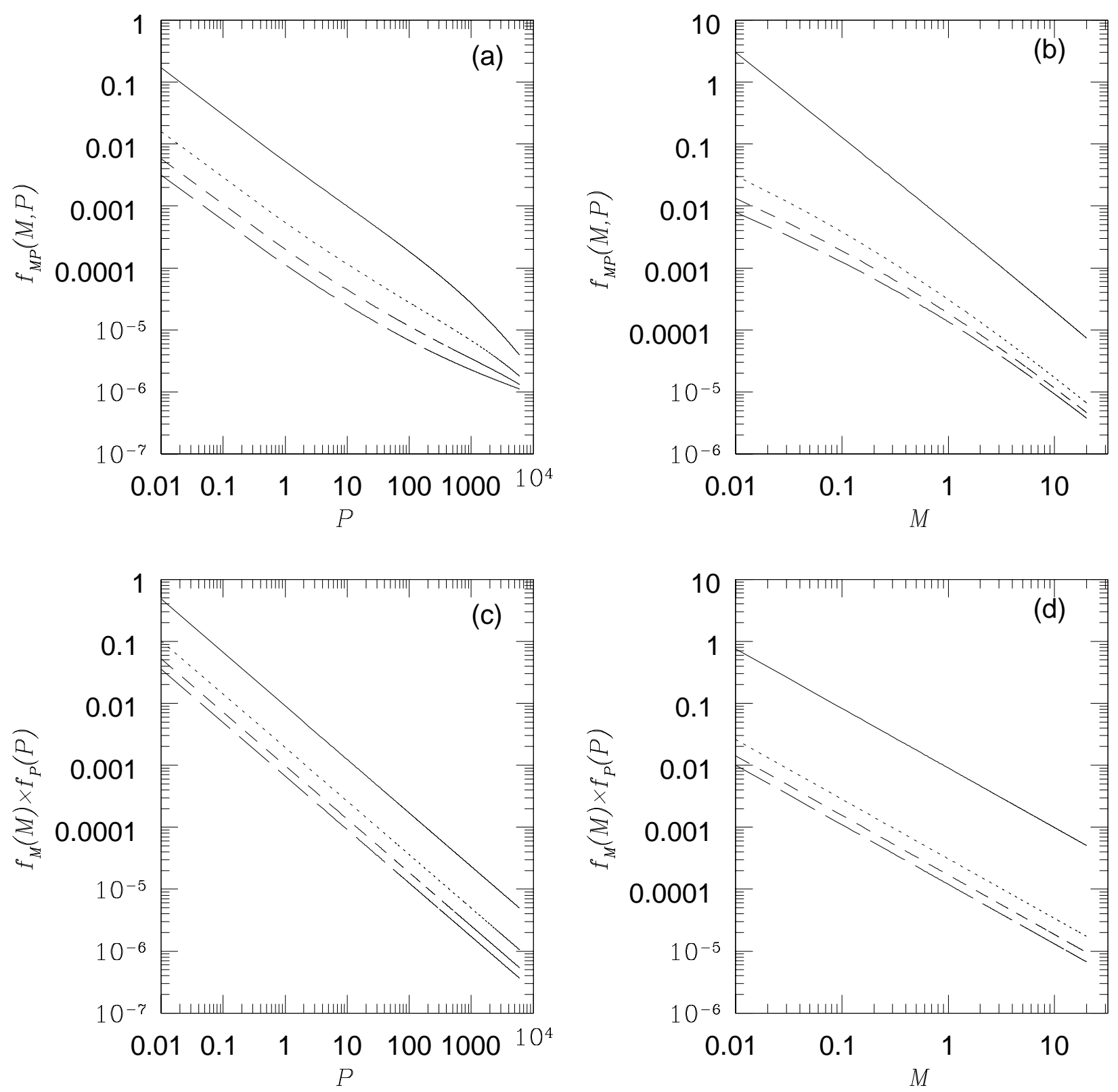

Fig. 9. - The mass and period functions in $M-P$ space for the results of Multiple Surveys. (a) The period functions of $M=1 M_{J}$ (solid curve), $M=5 M_{J}$ (dotted curve), $M=10 M_{J}$ (short dashed curve), and $M=15 M_{J}$ (long dashed curve). (b) The mass functions of $P=1$ day (solid curve), $P=50$ days (dotted curve), $P=100$ days (short dashed curve), and $P=150$ days (long dashed curve). (c) The independent period functions of $M=1 M_{J}$ (solid curve), $M=5 M_{J}$ (dotted curve), $M=10 M_{J}$ (short dashed curve), and $M=15 M_{J}$ (long dashed curve). (d) The independent mass functions of $P=1$ day (solid curve), $P=50$ days (dotted curve), $P=100$ days (short dashed curve), and $P=150$ days (long dashed curve). 